\title{
FPGA Implementation of a Clockless Stochastic LDPC Decoder
}

\author{
by \\ Christopher Ceroici
}

\author{
A thesis \\ presented to the University of Waterloo \\ in fulfillment of the \\ thesis requirement for the degree of \\ Master of Applied Science \\ in \\ Electrical and Computer Engineering
}

Waterloo, Ontario, Canada, 2014

(C) Christopher Ceroici, 2014 
I hereby declare that I am the sole author of this thesis. This is a true copy of the thesis, including any required final revisions, as accepted by my examiners. I understand that my thesis may be made electronically available to the public. 


\begin{abstract}
This thesis presents a clockless stochastic low-density parity-check (LDPC) decoder implemented on a Field-Programmable Gate Array (FPGA). Stochastic computing reduces the wiring complexity necessary for decoding by replacing operations such as multiplication and division with simple logic gates. Clockless decoding increases the throughput of the decoder by eliminating the requirement for node signals to be synchronized after each decoding cycle. With this partial-update algorithm the decoder's speed is limited by the average wire delay of the interleaver rather than the worst-case delay. This type of decoder has been simulated in the past but not implemented on silicon. The design is implemented on an ALTERA Stratix IV EP4SGX230 FPGA and the frame error rate (FER) performance, throughput and power consumption are presented for $(96,48)$ and $(204,102)$ decoders.
\end{abstract}




\section{Acknowledgements}

I would first like to thank my supervisor Dr. Vincent Gaudet for his guidance in this project.

I would also like to thank Brendan Crowley, Navid Bahrani and Manpreet Singh for their assistance with HDL and FPGA operation.

Thank you to the Natural Sciences and Engineering Research Council for funding and to ALTERA for donating the FPGA board. 


\section{Dedication}

This thesis is dedicated to my family for their support which made this possible. 


\section{Table of Contents}

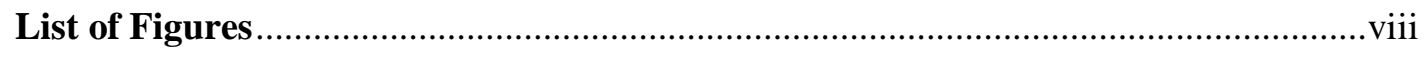

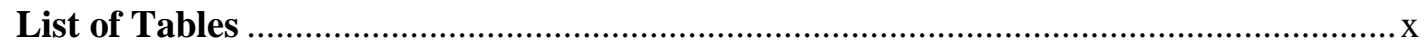

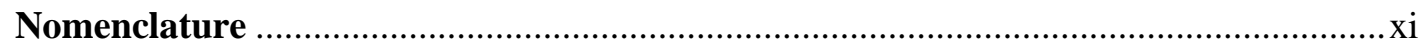

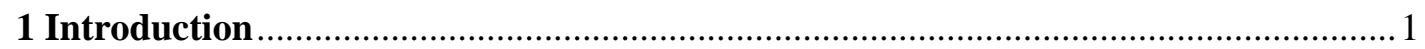

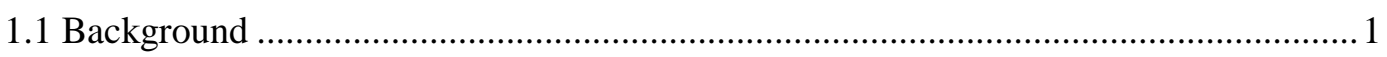

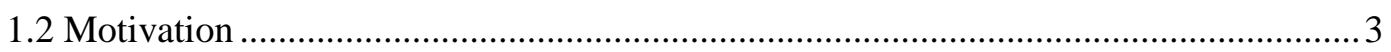

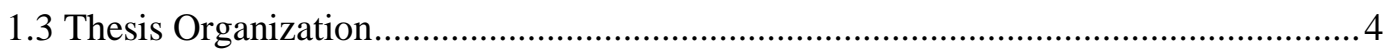

2 Background

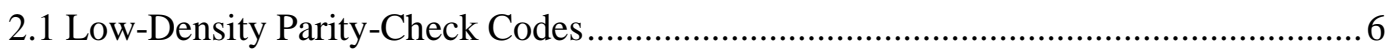

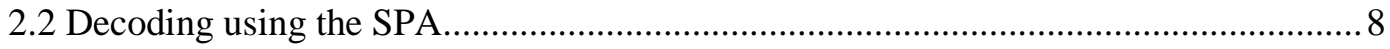

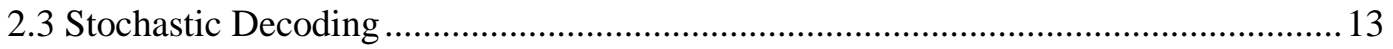

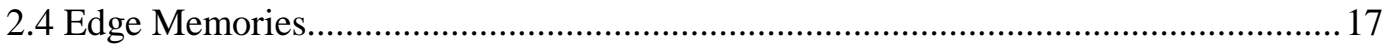

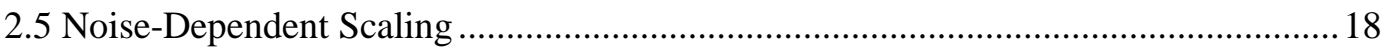

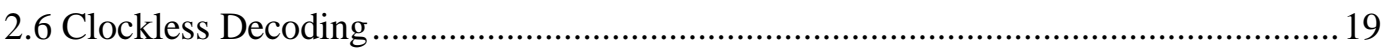

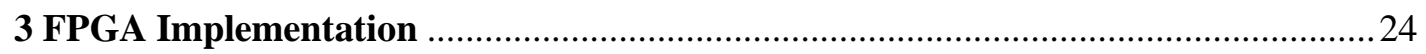

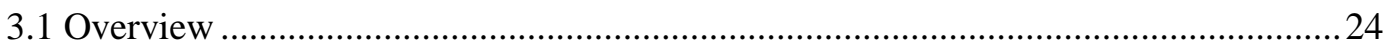

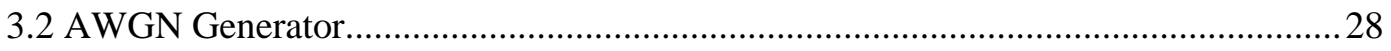

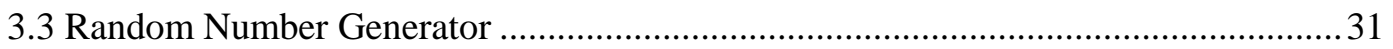


3.5 Controller

3.6 Variable Nodes

3.7 Parity-Check Nodes. 38

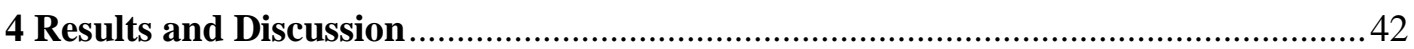

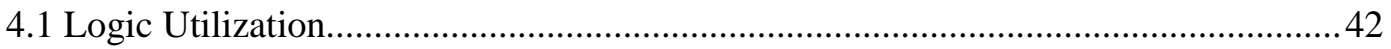

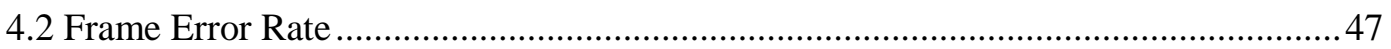

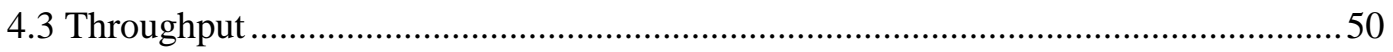

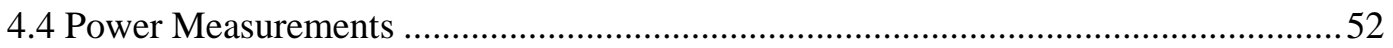

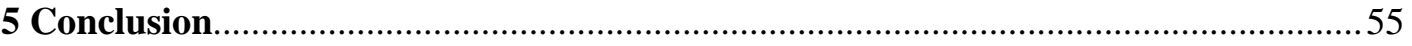

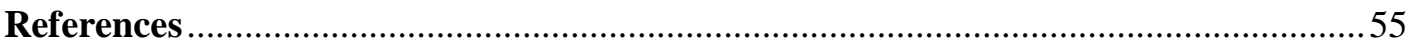




\section{List of Figures}

1.1 Block diagram of a communication system with channel coding . ................................... 2

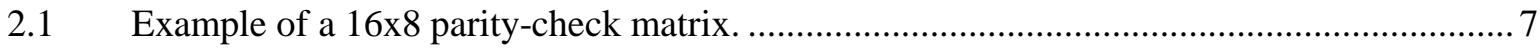

2.2 Tanner graph representation of the $(16,8)$ parity-check matrix from Figure $2.1 \ldots \ldots \ldots \ldots \ldots \ldots . .8$

2.3 Numerical simulation of the frame error rate (FER) of the $(16,8)$ parity-check matrix

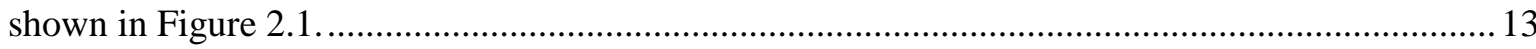

2.4 Stochastic gates for multiplication (top) addition (middle) and division (bottom). The output bits will approximate the calculated value with more accuracy as more bits are used.......... 14

2.5 Simple circuit implementation of a 2-input stochastic variable node. .............................. 16

2.6 Simple circuit implementation of a 2-input parity-check node.......................................... 16

2.7 Simple circuit implementation of a 2-input parity-check node. ......................................... 19

2.8 Simple Tanner graph demonstrating the effects of wire delays, shown in blue in

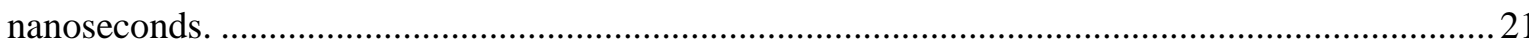

2.9 Simulation of the parity-check node signals of the Tanner graph in Figure using both a

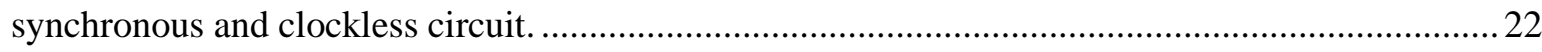

3.1 Block diagram of the clockless stochastic LDPC decoder.......................................... 27

3.2 Gaussian distributions generated from a Box-Muller numerical simulation, the proposed

Gaussian generation technique, and the scaled ideal probability distribution. ................................ 30

3.3 Illustration of the seed rotating technique. Each block represents a set of seeds used by

LFSRs. Arrows represent which block of seeds is currently being used. 
3.4 Comparator used for stochastic bit generation. The 8-bit inputs $\mathrm{P}_{\mathrm{i}}$ and $\mathrm{R}$ are the channel probabilities and a random number.

3.5 Circuit implementation of a 3-input clockless stochastic variable node using an edge memory $(\mathrm{EM})$ 37

3.6 Schematic of the edge memory (EM) used in the clockless stochastic variable node. ........ 38

3.7 Parity-check node circuit for a synchronous stochastic LDPC decoder. The red and blue

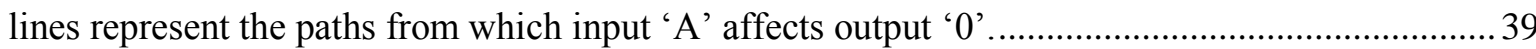

3.8 Circuit implementation of a 6-input clockless stochastic parity-check node...................... 40

4.1 Chip layout of $(96,48)$ decoder (left) and $(204,102)$ decoder (right). ............................... 44

4.2 Distribution of interleaver wire delays of the $(96,48)$ decoder. Delays were determined

by estimating the path delays of the synthesized design

4.3 Distribution of interleaver wire delays of the $(204,102)$ decoder. Delays were

determined by estimating the path delays of the synthesized design.

4.4 Frame error rate (FER) measurements of the $(96,48)$ clockless stochastic decoder implemented on an FPGA.

4.5 Frame error rate (FER) measurements of the $(96,48)$ clockless stochastic decoder implemented on an FPGA. Results are shown with noise-dependent scaling parameter $\mathrm{a}=2$. Measurements are compared with a numerical decoding simulation 50

4.6 Coded throughput measurements of the $(96,48)$ and $(204,102)$ clockless stochastic decoders implemented on an FPGA. 51

4.7 Power consumption of $(96,48)$ and $(204,102)$ decoders implemented on an FPGA...........53

4.8 Energy-per-coded bit of $(96,48)$ and $(204,102)$ decoders implemented on an FPGA. .........54 


\section{List of Tables}

3.1 Summary of the variable node behavior.........................................

4.1 Summary of the logic utilization of the $(96,48)$ and $(204,102)$ stochastic clockless

decoders synthesized on a ALTERA Stratix IV EP4SGX230 FPGA........................43 


\section{Nomenclature}

\begin{tabular}{ll} 
AWGN & Additive White Gaussian Noise \\
BER & Bit Error Rate \\
FER & Frame Error Rate \\
GF & Galois Field \\
H & Parity-Check Matrix \\
LDPC & Low-Density Parity-Check \\
LFSR & Linear Feedback Shift Register \\
LLR & Log Likelihood Ratio \\
LUT & Lookup Table \\
PDF & Probability Density Function \\
RN & Random Number \\
& \\
\hline SN & Sool command language \\
&
\end{tabular}




\section{Chapter 1}

\section{Introduction}

\subsection{Background}

Channel coding is a technique used in digital communications to minimize the rate of errors when messages are transmitted over a noisy channel. Figure 1.1 illustrates a high level view of a baseband communication system where messages are encoded at the transmitter end and then enter a channel where noise is superimposed on the encoded message. At the receiver end, the decoder attempts to recover the initial message and minimize errors introduced by the noisy channel. Channel coding is done by adding controlled redundant parity-check symbols to a signal prior to transmission, allowing the decoder to reconstruct the original message with high reliability at the receiver end. 


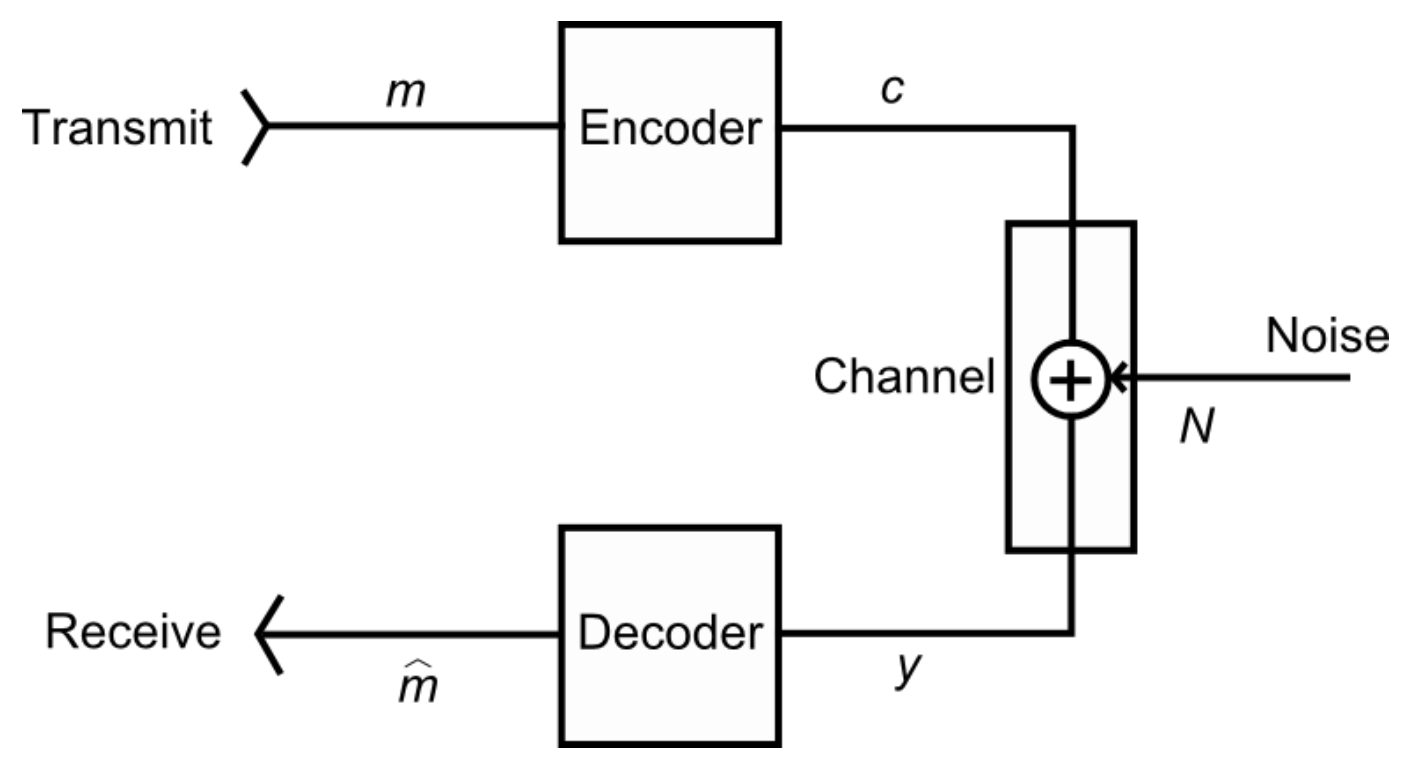

Figure 1.1: Block diagram of a communication system with channel coding.

Low-Density Parity-Check (LDPC) codes are a family of forward error control codes first proposed by Gallager in his $\mathrm{PhD}$ thesis in 1960 and further developed in the following years [1,2]. An LDPC code is represented by a parity-check matrix $\mathbf{H}$ that has a low density of nonzero elements. This thesis focuses on the binary case where the only elements in $\mathbf{H}$ are '1's and '0's. The matrix $\mathbf{H}$ can be represented by a Tanner graph, a network of two types of nodes, parity-check nodes and variable nodes, which exchange signals during the decoding process. The nonzero elements of $\mathbf{H}$ dictate the connections between these nodes in a network of wires known as the interleaver. Due to the computing requirements for decoding, LDPC codes were not used widely following their discovery and were almost completely forgotten. Turbo codes [3], another type of error correcting code, were discovered in 1993 by Berrou et al. and were found to approach the Shannon capacity limit, the theoretical maximum rate at which 
information can be transmitted over a noisy communications channel. In 1997, LDPC codes were rediscovered by MacKay et al. [4] and through the use of a probabilistic decoder rivaled the performance of Turbo codes [4,5]. Following this discovery, the field of LDPC codes has grown substantially.

The Sum-Product Algorithm (SPA) is an iterative decoding process which approximates a maximum likelihood decoder but it more suitable for circuit implementations [6-10]. Due to the many multiplication and division operations in the SPA, LDPC decoders usually operate in the logarithmic domain which converts these multiplication operations into summations. The SPA has a high degree of parallelism, so to maximize throughput in highspeed applications, parallel LDPC decoder implementations are common [11].

\subsection{Motivation}

Stochastic computing is a method of random numerical computation first proposed in 1953 by von Neumann [40]. Stochastic computing utilizes uncertainties and probabilities to perform useful calculations. Continuous values can be represented by streams of random bits which often results in lower complexity circuits. A decoding method which relies of the principles of stochastic computing was proposed by Gaudet et al. in [41] as an alternative to the SPA. This type of decoder benefits from lower complexity of the interleaver, however due to the serial nature of stochastic computing, the calculations within individual nodes must be done serially, reducing the throughput. To offset this deficiency, higher clock frequencies are often used. However, a large high-speed clock network results in high power dissipation due to the high 
switching activity of the clock. Furthermore, longer wires are required to meet the challenges of the routing constraints of the LDPC interleaver, limiting the clock speed and hence the throughput. Various techniques have been proposed to circumvent this problem such as asynchronous decoding [12,13] and clockless decoding [14,15].

In a clockless decoder, the communication across the interleaver is restricted only by the wiring delay. Furthermore, the decoding process no longer waits for all node calculations to be completed before beginning the next decoding cycle. Since the wire delays will vary across the interleaver in this continuous decoding technique, the decoding speed is limited by the average of the wire delays rather than the largest wire delay. This type of decoder has been demonstrated in high-level simulations in [14].

This thesis presents a Field-Programmable Gate Array (FPGA) implementation of a clockless stochastic LDPC decoder and corresponding performance measurements. This work demonstrates the feasibility for a practical clockless stochastic decoder.

\subsection{Thesis Organization}

This thesis is organized as follows: Chapter 2 reviews the sum-product algorithm (SPA) as well as LDPC codes, stochastic decoders, and their synchronous, asynchronous and clockless implementations. Chapter 3 presents the design of a clockless stochastic LDPC decoder with details of individual components. Chapter 4 reports the frame error rate, throughput, and power performance of the decoder and discusses some trade-offs between them. The FPGA logic 
utilization is also discussed in this chapter. Chapter 5 discusses possible future work and applications of this decoder and concludes the thesis. 


\section{Chapter 2}

\section{Background}

This chapter summarizes the concepts of LDPC codes, Tanner graphs and the sum product algorithm in Sections 2.1 and 2.2. Stochastic decoding is introduced in section 2.3 while sections 2.4 and 2.5 present the concepts of noise-dependent scaling and edge memories. Section 2.6 summarizes clockless stochastic decoding of LDPC codes and its benefits.

\subsection{Low-Density Parity-Check Codes}

Discovered in the early 1960s [1,2], LDPC codes are a class of linear block codes with a sparse parity-check matrix, $\boldsymbol{H}$ of size $(n-k)$ by $n[37,38]$. LDPC codes can be defined for any order of Galois field but this thesis will focus on LDPC codes over GF(2). The length of the message is $k$ symbols (usually bits) while the encoded message is of length $n$. LDPC codes have a code rate of $R=k / n$ which represents the ratio of message bits to codeword bits. A binary vector $\boldsymbol{c}$ is a codeword if $\boldsymbol{c} \boldsymbol{H}^{\boldsymbol{T}}=\mathbf{0}$. In other words, a codeword must be orthogonal to every row of $\boldsymbol{H}$. There also exists a generator matrix $\boldsymbol{G}$ consisting of the basis vectors of the decoder and from 
which $\boldsymbol{G} \boldsymbol{H}^{\boldsymbol{T}}=\mathbf{0}$. The generator matrix can be used to encode messages and is defined in terms of $\boldsymbol{H}$ from the expressions $\boldsymbol{H}=\left[-\boldsymbol{P}^{\boldsymbol{T}} \mid \boldsymbol{I}_{\boldsymbol{n}-\boldsymbol{k}}\right]$ and $\boldsymbol{G}=\left[\boldsymbol{I}_{\boldsymbol{k}} \mid \boldsymbol{P}\right]$.

An LDPC code can also be displayed graphically as a bipartite Tanner Graph [16].

Within a Tanner Graph there exists two types of nodes: variable nodes (shown as circles) and parity-check nodes (shown as squares). A line connecting a variable node to a parity-check node is represented by a ' 1 ' in $\boldsymbol{H}$. The variable nodes each represent a single codeword bit while each parity-check node represents a single parity-check equation. A line connecting a variable node to a parity-check node suggests that this bit is included in that particular parity-check equation. An example block code with $n=16$ and $k=8$ is shown in Figure 2.1 with its corresponding Tanner Graph shown in Figure 2.2. The parity-check matrix notation $(k, n-k)$ denotes a Tanner graph with 'a' variable nodes and 'b' parity-check nodes.

$$
H=\left[\begin{array}{llllllllllllllll}
0 & 1 & 1 & 0 & 0 & 1 & 0 & 0 & 0 & 1 & 0 & 1 & 0 & 1 & 0 & 0 \\
0 & 1 & 0 & 1 & 0 & 0 & 0 & 1 & 0 & 0 & 1 & 0 & 1 & 0 & 0 & 1 \\
1 & 0 & 1 & 0 & 1 & 1 & 0 & 0 & 0 & 0 & 0 & 1 & 1 & 0 & 0 & 0 \\
0 & 1 & 0 & 0 & 0 & 0 & 0 & 1 & 0 & 0 & 1 & 0 & 0 & 1 & 1 & 1 \\
1 & 0 & 0 & 1 & 1 & 0 & 1 & 0 & 1 & 1 & 0 & 0 & 0 & 0 & 0 & 0 \\
0 & 0 & 0 & 0 & 1 & 0 & 1 & 0 & 1 & 0 & 0 & 1 & 1 & 0 & 1 & 0 \\
1 & 0 & 0 & 1 & 0 & 0 & 0 & 1 & 0 & 1 & 1 & 0 & 0 & 0 & 1 & 0 \\
0 & 0 & 1 & 0 & 0 & 1 & 1 & 0 & 1 & 0 & 0 & 0 & 0 & 1 & 0 & 1
\end{array}\right]
$$

Figure 2.1: Example of a $(16,8)$ parity-check matrix. 


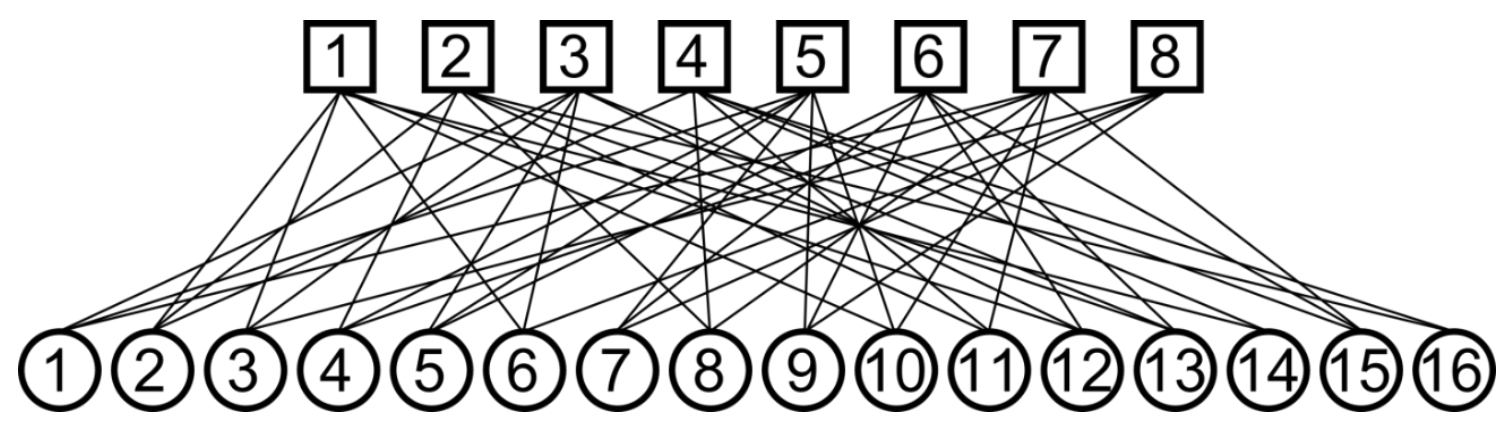

Figure 2.2: Tanner graph representation of the $(16,8)$ parity-check matrix from Figure 2.1.

\subsection{Decoding using the SPA}

There are two main types of decoding algorithms: hard-decision decoders and soft-decision decoders. Referring to Figure 1.1, the decoding process starts when a message vector $m$ is encoded to a code vector $c$ and transmitted through a channel. The code vector received from the channel, $y$, is equal to the original codeword with the addition of noise from the channel: $y=c+N$. The noise, $N$, can be modeled mathematically as AWGN. A binary hard-decision decoder begins by calculating the probability $p$ that individual bits of $y$ are either ' 1 ' or ' 0 '. The received codeword is then defined through hard decision making (e.g., if a symbol has a 0.55 probability of being ' 1 ', or any probability greater than 0.5 , then it is assumed to be ' 1 '). The hard-decision decoder then attempts to decode the original message by evaluating the paritycheck equations defined in $\mathbf{H}$ to determine if the received codeword is a valid codeword, and if not, which bits are erroneous. A soft-decision decoder operates on the probabilities that particular bits are either ' 0 ' or ' 1 ' and with knowledge of the channel probability distribution function (PDF) uses information from the parity-check bits to produce a more accurate 
estimation of the bit-value probabilities. In an iterative decoder, the hard decision bits can be calculated after each iteration to check for a valid codeword, otherwise the decoder continues to attempt to produce more accurate probabilities.

The Sum-Product algorithm (also known as belief propagation or the message passing algorithm) is an iterative soft-decision decoder. The goal of the Sum-Product Algorithm (SPA) is to calculate the probability that any given bit in the transmitted codeword $\overrightarrow{\boldsymbol{c}}=$ $\left(c_{0}, c_{1}, c_{2}, \ldots c_{n-1}\right)$ is equal to ' 1 ' given the received codeword $\overrightarrow{\boldsymbol{y}}=\left(y_{0}, y_{1}, y_{2}, \ldots y_{n-1}\right)$. A channel probability $P_{i}$ is received by each variable node calculated from $y_{i}$ using the equation (for the additive white Gaussian noise (AWGN) channel) [16]:

$$
P_{i}=\frac{1}{1+\exp \left(\frac{-2 y_{i}}{\sigma}\right)}
$$

where $\sigma$ is the noise variance. The variable node $i$ then sends a probability $q_{i j}$ (which is equal to $P_{i}$ for the first iteration) to each connected parity-check node $j$ which then calculates a new probability $r_{j i}$ to update all connected variable nodes from its parity-check equation

$$
r_{j i}=\frac{1}{2}+\frac{1}{2} \prod_{i^{\prime}}\left(1-q_{i^{\prime} j}\right)
$$


where $i^{\prime}$ are all the variable nodes $\left(i_{0}, i_{1}, i_{2} \ldots\right)$ connected to parity-check node $j$ with the exception of $i$, the node being updated. The connected parity-check nodes are then updated using the equations:

$$
\begin{gathered}
q_{i j}(1)=K_{i j} P_{i} \prod_{j^{\prime}} r_{j^{\prime} i} \\
q_{i j}(0)=K_{i j}\left(1-P_{i}\right) \prod_{j^{\prime}}\left(1-r_{j^{\prime} i}\right)
\end{gathered}
$$

where $K_{i j}$ is a normalization constant selected such that $q_{i j}(1)+q_{i j}(0)=1$. Note that only $q_{i j}(1)$ is used in the parity-check node equation. At each iteration, the probability that any particular bit is equal to ' 1 ' or ' 0 ', or the hard decision bit, can be calculated using:

$$
\begin{gathered}
Q_{i}(1)=K_{i} P_{i} \prod_{j} r_{j i} \\
Q_{i}(0)=K_{i}\left(P_{i}-1\right) \prod_{j} r_{j i}
\end{gathered}
$$

where the product is computed across all parity-check nodes $j$ connected to variable node $i$. $Q_{i}(1)$ and $Q_{i}(0)$ are the probabilities that codeword $i$ is equal to ' 1 ' or ' 0 ', respectively, and $K_{i}$ is a normalization constant selected such that $Q_{i}(1)+Q_{i}(0)=1$. This process is continued until either the hard decision bits form a valid codeword or until a maximum number of 
iterations has been completed. If the decoder reaches the maximum number of iterations an error is declared and the bit error rate can be computed by comparing the hard decision bits with the original codeword.

The SPA involves many multiplication and division operations for both node computations and for the normalization conditions of the variable nodes. Therefore, a hardware implementation of such a decoder would be extremely area inefficient as hardware multipliers are much more complex than hardware adders. To bypass this problem, hardware SPA decoders operate in the logarithm domain. Rather than calculating the channel probability $P_{i}$ initially, a Log-Likelihood Ratio (LLR) $L_{i}=\log \left(\frac{P\left(y_{i}=0\right)}{P\left(y_{i}=1\right)}\right)$ is calculated. For an AWGN channel, this is calculated using the following equation.

$$
L_{i}=\frac{2 y_{i}}{\sigma^{2}}
$$

The variable and parity-check node likelihood signals are then updated using the following equations.

$$
\begin{gathered}
L\left(r_{j i}\right)=\left[\prod_{i^{\prime}} \operatorname{sign}\left(L\left(q_{i^{\prime} j}\right)\right)\right] \cdot \phi\left(\sum_{i^{\prime}} \phi\left(\left|L\left(q_{i^{\prime} j}\right)\right|\right)\right) \\
\phi(x)=\log \left(\frac{e^{x}+1}{e^{x}-1}\right) \\
L\left(q_{i j}\right)=L_{i}+\sum_{j^{\prime}} L\left(r_{j^{\prime} i}\right)
\end{gathered}
$$


$L\left(r_{j i}\right)$ is the log-likelihood ratio signal sent from parity-check nodes to variable

nodes and $L\left(q_{i j}\right)$ is the ratio sent from variable to parity-check nodes. The hard decision bits can be calculated using:

$$
L\left(Q_{i j}\right)=L_{i}+\sum_{j} L\left(r_{j i}\right)
$$

Where bit $i$ is equal to ' 1 ' if $L\left(Q_{i j}\right)<0$ and is equal to ' 0 ' otherwise. This is the same equation used for calculating $L\left(q_{i j}\right)$ except signals from all of the connected nodes are considered. The log-domain SPA can be implemented in a circuit much more efficiently than the SPA since the multiplication operations have been reduced to summation operations. The calculation of $\phi(x)$ can be done using a look-up table (LUT) efficiently since it is an even function [42]. An additional LUT is required for the initial logarithm transformation of the received channel probability. A simulation of the block code from Figure 2.1 is shown in Figure 2.3 using the log-domain SPA Equations 2.7 to 2.11 . Note that this decoder is used as a demonstration and a practical LDPC decoder would use a much larger code resulting in much better performance. Figure 2.3 shows the frame error rate (FER), or the fraction of errors that were detected at varying signal-to-noise ratios (SNRs) through an entire decoding process after repeating the simulation many times until a significant number of errors have been detected. This simulation was calculated using AWGN applied to zero-value codewords. 


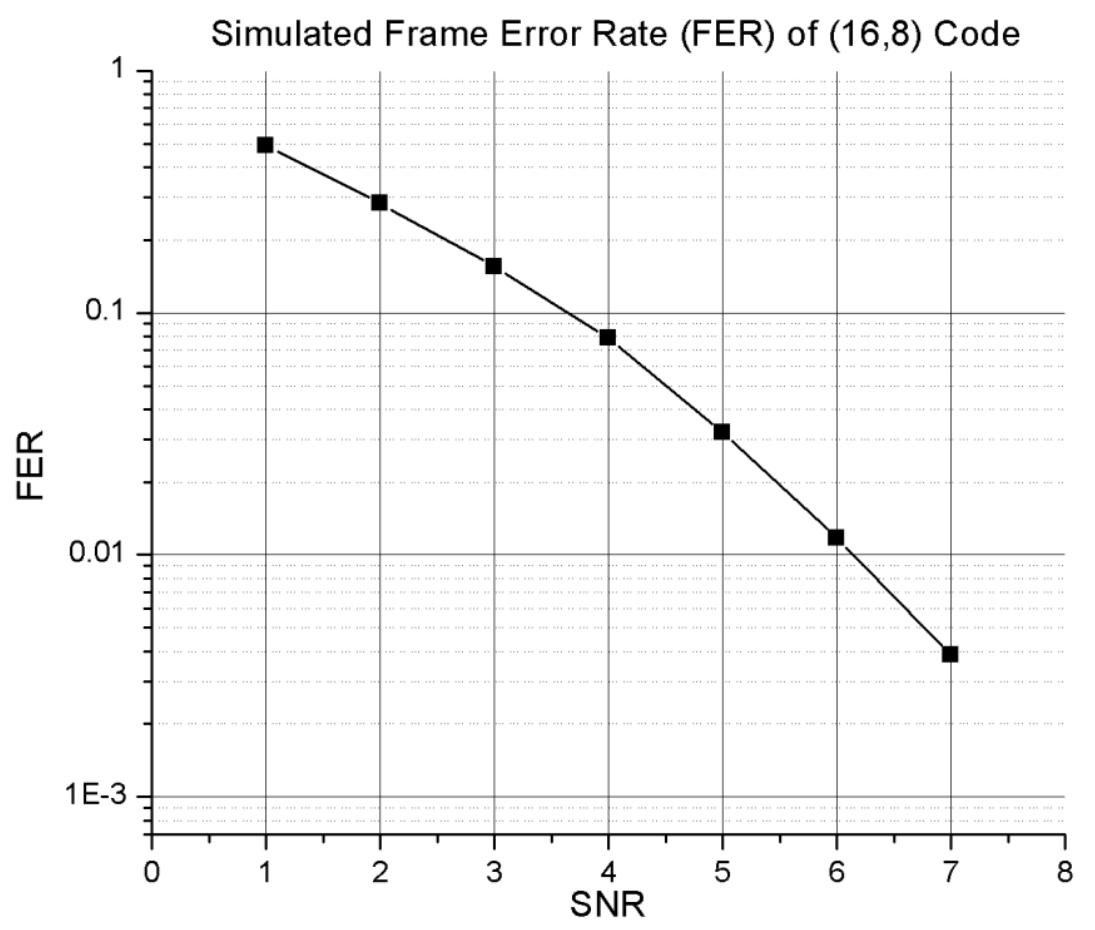

Figure 2.3: Numerical simulation of the frame error rate (FER) of the $(16,8)$ parity-check matrix shown in Figure 2.1.

\subsection{Stochastic Decoding}

Stochastic computing is the process of using randomness to achieve useful computation.

Continuous value signals can be represented by a stream of randomly generated bits where the values are communicated from the statistical means of the bit streams. The probability of 
stochastic stream is equal to the ratio of ' 1 's to ' 0 's and so there are many possible

representations of any probability. For example the probability 0.6 can be represented by the streams 10110 or 0110101011 . The order in which the ' 1 's appear in the stochastic sequence is not important.

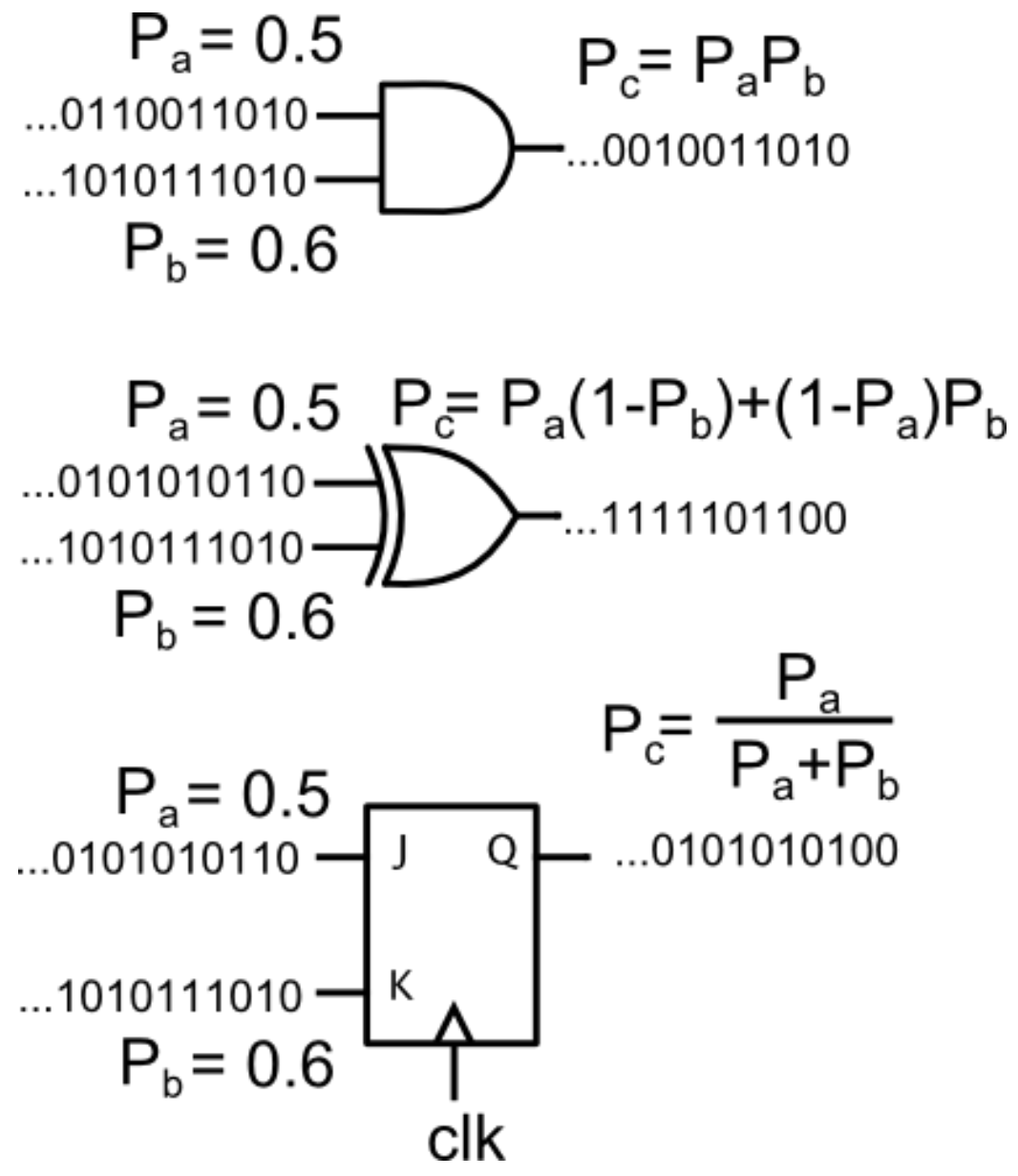

Figure 2.4: Stochastic gates for multiplication (top) soft XOR (middle) and division (bottom). The output bits will approximate the calculated value with more accuracy as more bits are used. 
Many operations have simple equivalent single-gate stochastic representations $[17,18]$, illustrated in Figure 2.4. The multiplication of two probabilities, such as that in Equation 2.3, has an equivalent stochastic operation, a bitwise AND gate. Similarly, two probabilities can undergo a soft XOR operation through the use of an XOR gate while division operations, such as that of the normalization condition required to solve Equation 2.4, are equivalent to a J-K flip flop in the stochastic domain. Stochastic division contains a memory element which emphasizes the fact that stochastic computation is a serial process. Stochastic addition and subtraction of probabilities should be normalized since they are not closed operations. A probability can be inverted with the use of a single inverter gate to calculate $P_{0}=1-P_{1}$. These stochastic gate representations are easier to implement in digital hardware and so stochastic decoders benefit from the decreased wiring complexity [27-33]. In addition, only a single wire is required to communicate a stochastic signal. The SPA equations can therefore be re-written in the stochastic domain. The equation for a variable node with two inputs, $P_{a}$ and $P_{b}$, and output $P_{C}$ is:

$$
P_{c}=\frac{P_{a} P_{b}}{P_{a} P_{b}+\left(1-P_{a}\right)\left(1-P_{b}\right)}
$$

Figure 2.5 shows a stochastic digital circuit implementation of a variable node. Note that a J-K flip flop is required for the normalization component of Equation 2.4 meaning that variable node computations will have a memory. This is expected since a stochastic stream is defined in terms of the serial arrangement of bits. 


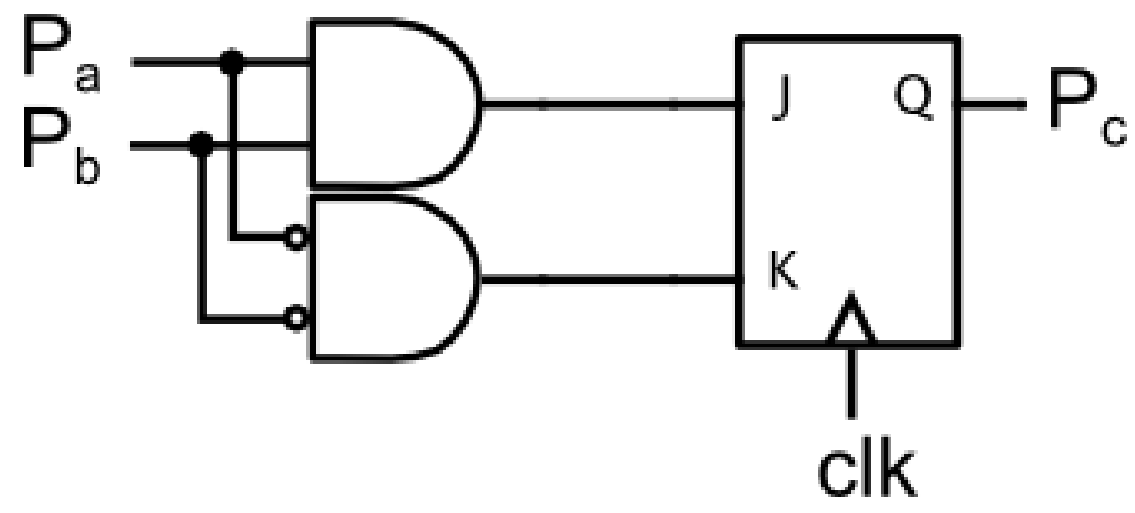

Figure 2.5: Simple circuit implementation of a 2-input stochastic variable node.

Since the parity-check node equation is simply an addition, its stochastic equivalent is just an XOR operation between its inputs.

$$
P_{c}=P_{a}\left(1-P_{b}\right)+P_{b}\left(1-P_{a}\right)
$$

A basic two-input parity-check node is shown in Figure 2.6.

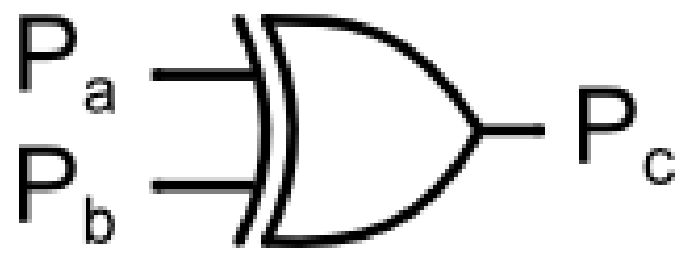

Figure 2.6: Simple circuit implementation of a 2-input parity-check node.

The decoding process starts with initializing the J-K flip-flops in the variable node with stochastic bits from the received channel probabilities. Following this, at every clock edge both 
sets of nodes are updated with signals from their connected nodes. The decoding process continues until either a valid codeword is found, identified by the parity-check equations at the parity-check nodes being satisfied, or until a pre-defined number of clock cycles has completed at which point an error is declared and the next frame begins.

\subsection{Edge Memories}

The variable node circuit shown in Figure 2.5 is not ideal since it can result in locked states during the decoding process. Locked states are cases where the output of the node remains the same over several decoding cycles. This lack of switching activity can slow the convergence of the decoder. Tanner graphs with cycles are particularly susceptible to this phenomenon. A cycle can result in a particular group of nodes becoming locked in a fixed state [33]

To circumvent this problem edge memories (EMs) were proposed by Tehrani et al. in [31]. EMs are memory elements, usually shift registers, which are used instead of J-K flip-flops and update their values based on the inputs of the variable node. The purpose of EMs is to rerandomize stochastic streams so that consecutive bits are independent of each other. The output bit of the variable node can be classified as either regenerative or degenerative. A regenerative bit is the case where the inputs to a variable node are equal. A degenerative bit is produced when the node inputs are not equal. If the variable node produces a regenerative bit it is used as the output of the node and also stored in the EM. When a degenerative bit is produced it is ignored and the output of the variable node is instead selected from the EM at a random address. This mechanism prevents the decoder from becoming stuck in a "lock" state where there is no 
switching activity at a variable node for a period of time. The EM must also have a forgetting mechanism where new regenerative bits replace old ones. EMs are usually preloaded with bits from the channel probability prior to decoding. Decoding can also begin with the EMs initialized to a zero state at the cost of slower decoder convergence.

\subsection{Noise-Dependent Scaling}

As the SNR changes, so will the amount of switching activity. This is because the channel probabilities will not use the full scale. Less switching activity results in slower convergence and poorer FER performance. Noise-dependent scaling (NDS) is a method of ensuring a constant amount of switching activity across different SNRs [31]. This is done by scaling the received LLRs by a parameter proportional to the SNR. The scaled LLR $L_{i}^{\prime}$ is calculated using:

$$
L_{i}^{\prime}=\left(\frac{2 \alpha \sigma^{2}}{Y}\right) L_{i}=\frac{4 \alpha y_{i}}{Y}
$$

where $L_{i}$ is the LLR calculated using Equation 2.7. The parameter $\alpha$ is chosen experimentally for best performance. $Y$ is set to be the maximum expected unscaled LLR value, usually set to $Y=6$. Notice that the $\sigma$ cancels in the above equation. This suggests that the noise power will have no effect on the magnitude of $L_{i}{ }^{\prime}$ and therefore the switching activity. However, in a practical decoder the LLR value $L_{i}$ is usually the decoder input so a channel noise measurement will still be required to obtain $\sigma$. This scaling operation is usually done using a LUT. 


\subsection{Clockless Decoding}

Two alternatives to synchronous decoding are asynchronous decoding and clockless decoding [12-15]. Asynchronous decoding eliminates the global clock and instead uses a handshaking mechanism for nodes to communicate across the interleaver. Using these request-acknowledge signals rather than a large clock network, as illustrated in Figure 2.7, eliminates the problem of varying clock delays across different nodes. This increases the overall throughput as well as reduces power consumption. However the introduction of request-acknowledge signals does incur a cost as there is an increase in the size and wiring complexity of the interleaver.

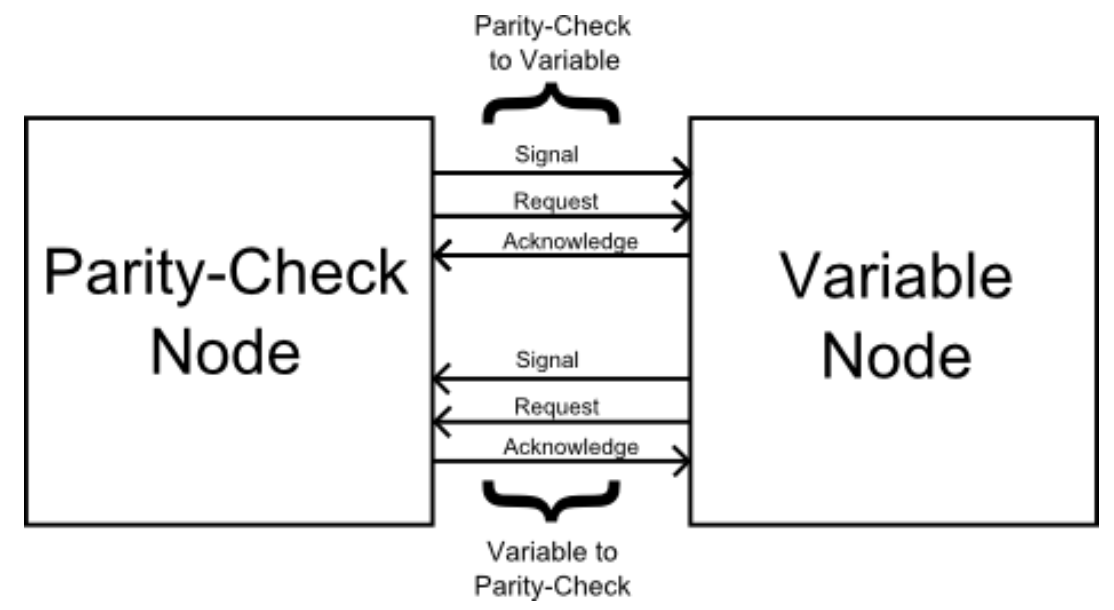

Figure 2.7: Simple circuit implementation of a 2-input parity-check node.

When a parity-check node finishes its calculation it must wait for every other paritycheck node to complete as well before updating the variable nodes. Since the wiring delay between different nodes will vary, the speed of the decoder will be limited by the largest wire delay in the interleaver. 
If a node were to send its calculated bit immediately to its connected nodes rather than waiting, some of the inputs of the connected nodes would be new while others would be outdated. However, since stochastic computations depend on the time averages of binary signals the intermediate bit produced by a node with outdated inputs doesn't have a significant impact on the overall decoding process. Ignoring this synchronization constraint eases the restrictions on the decoder design and allows for the exploration of larger design spaces than previously possible. Clockless decoding removes these two limitations by ignoring the synchronization constraint amongst nodes. As soon as a node has completed its calculation it immediately sends its output to the connected nodes. This partial-update algorithm, described in [19-21], allows for the decoding speed to be determined by the average wire delay. A sample Tanner graph is shown in Figure 2.8 with illustrated wire delays shown in blue in nanoseconds, for illustrative purposes. 


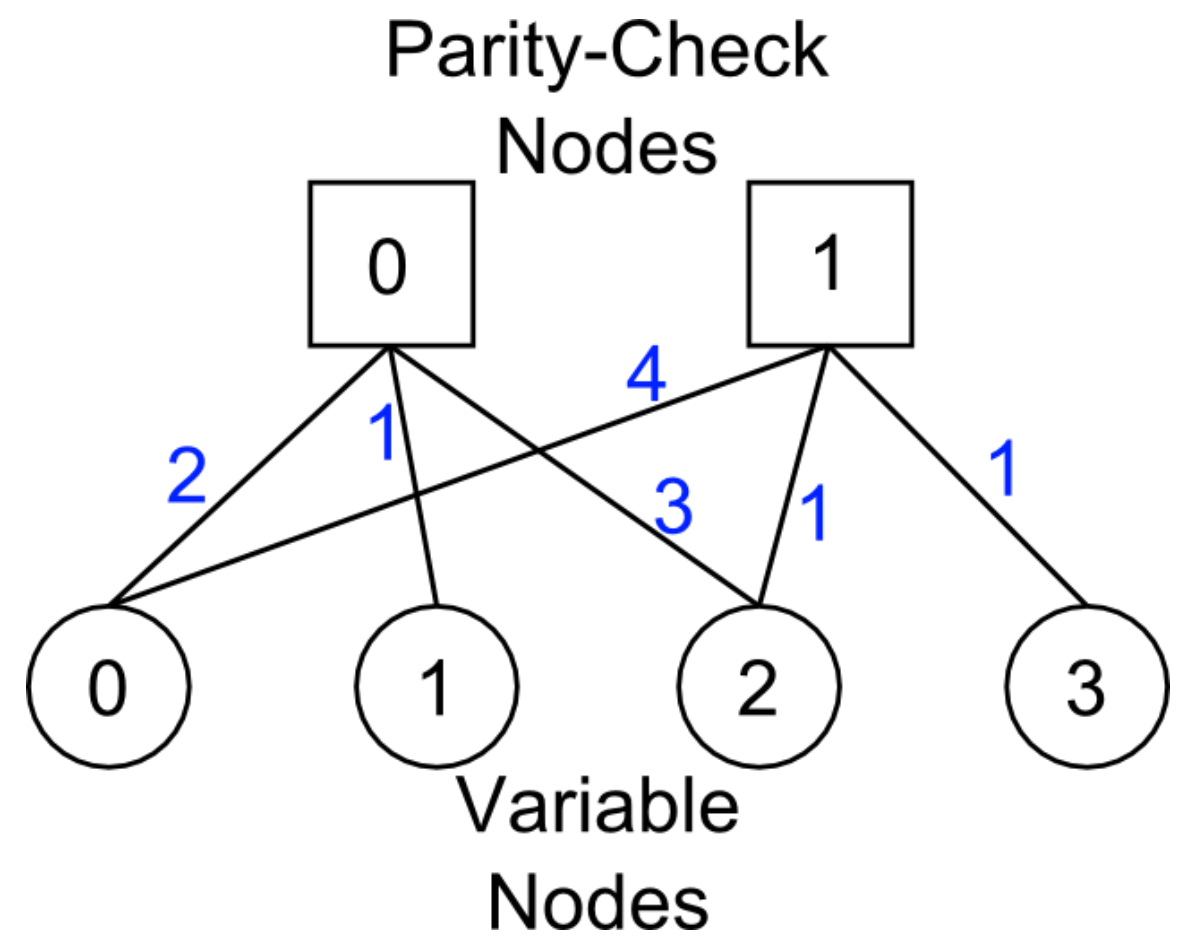

Figure 2.8: Simple Tanner graph demonstrating the effects of wire delays, shown in blue in nanoseconds.

If a synchronous decoder were used for this graph the throughput would be restricted by the largest wire delay, the delay between parity-check node 1 and variable node 0 , while a clockless decoder would not. This can be seen in Figure 2.9 which shows a simulated set of communications between the nodes of Figure 2.8 for both a synchronous decoder and a clockless decoder with their respective wire delays. The nodes in this simulation are the simplest stochastic variable and parity-check node designs shown in Figures 2.5 and 2.6. Here, the synchronous decoder uses the maximum clock frequency allowed by the wire delays. 


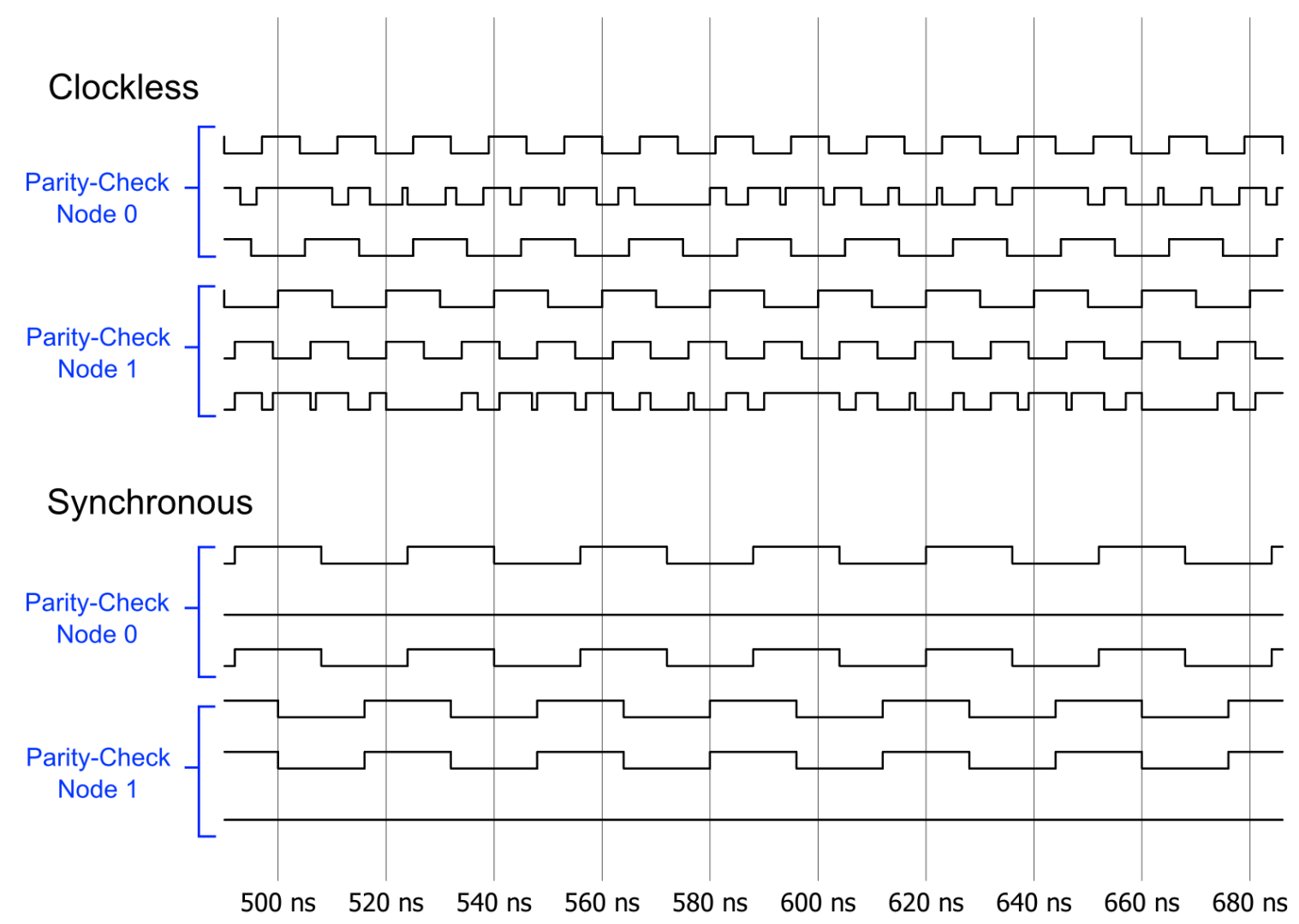

Figure 2.9: Simulation of the parity-check node signals of the Tanner graph in Figure 2.8 using both a synchronous and clockless circuit.

Comparing the first signal of parity-check node 0 (the connection from parity-check node 0 to variable node 0 ) of the synchronous and clockless decoders in Figure 2.9, the synchronous signal has a period of $31.5 \mathrm{~ns}$ while the clockless signal has a period of $14 \mathrm{~ns}$. The ratio of these periods, 2.25 , is approximately equal to 2 , the ratio of the largest wire delay to the average wire delay. Because of the different wire delays the nodes of the clockless decoder will often use outdated signals for its computations resulting in more switching activity. However, 
these extra signals do not have a significant negative effect on the calculations or the speed of convergence. A stochastic LDPC decoder using the partial-update scheduling algorithm was simulated in [15] using $90 \mathrm{~nm}$ technology and resulted in a lower error floor, the flattening of the error rate curve at high SNR, than a synchronous decoder. There has been no physical demonstration of this type of decoder to date. The goal of this thesis is to demonstrate a proofof-concept design of a clockless stochastic LDPC decoder and to learn as much as possible from this implementation. 


\section{Chapter 3}

\section{FPGA Implementation}

This chapter describes the architecture of clockless stochastic LDPC decoder. Section 3.1 summarizes the overall design. Section 3.2 and 3.3 presents the designs of an AWGN generator and the (pseudo) random number generators required for real-time testing of the decoder hardware. Section 3.4 describes the comparator used for generating stochastic bits. The designs of the variable and parity-check nodes are presented in Sections 3.6 and 3.7.

\subsection{Overview}

The clockless stochastic LDPC decoder was implemented on an ALTERA Stratix IV EP4SGX230 FPGA using the Quartus II set of computer aided design tools [36]. The design consists of the following modules:

- An AWGN generator which (pseudo) randomly generates zero-codeword LLRs and then scales them according to the noise power.

- A comparator used for the conversion of LLRs into stochastic streams.

- A controller to initialize the decoder and to begin and terminate frames. 
- The parity-check and variable nodes and the interleaver connecting them.

A block diagram of the decoder is shown in Figure 3.1. At each initialization phase LLRs are generated and scaled. The LLRs are then converted into a stochastic stream using an external or local clock. These stochastic bits are then passed on to the variable nodes for decoding or preloading the EMs during initialization. This initialization phase could be skipped by preloading the EMs with '0's at the expense of the decoder's speed of convergence.

However, since this decoder uses zero-value codewords for testing purposes the variable nodes would begin decoding with a bias towards the correct codeword. The EMs in the variable nodes use random addresses generated from LFSRs. The decoding process terminates when a valid codeword is detected by the variable nodes or until the controller declares an error.

The Verilog hardware description files (HDL) for the interleaver as well as any other modules dependent on $\boldsymbol{H}$ are generated using a $\mathrm{C}++$ script which reads in $\boldsymbol{H}$ in alist format, an efficient way of representing large, sparse binary matrices. $\boldsymbol{H}$ is then converted into matrix form where the ' 1 's indicate connections between the variable and parity-check nodes. This matrix is then used to generate the HDL for the interleaver, the module where the variable and paritycheck node modules are instantiated and their connecting wires are assigned according to $\boldsymbol{H}$. The script also generates sets of (pseudo) random seeds to initialize the linear feedback shift registers (LFSRs).

The $96 \times 48$ and 204x102 parity-check matrices used for the designs in this thesis research were acquired from [22]. However, the script used here can generate HDL files for any 
regular $(3,6)$ LDPC code, or any code with a Tanner graph having parity-check nodes with six connections and variable nodes with three connections. 


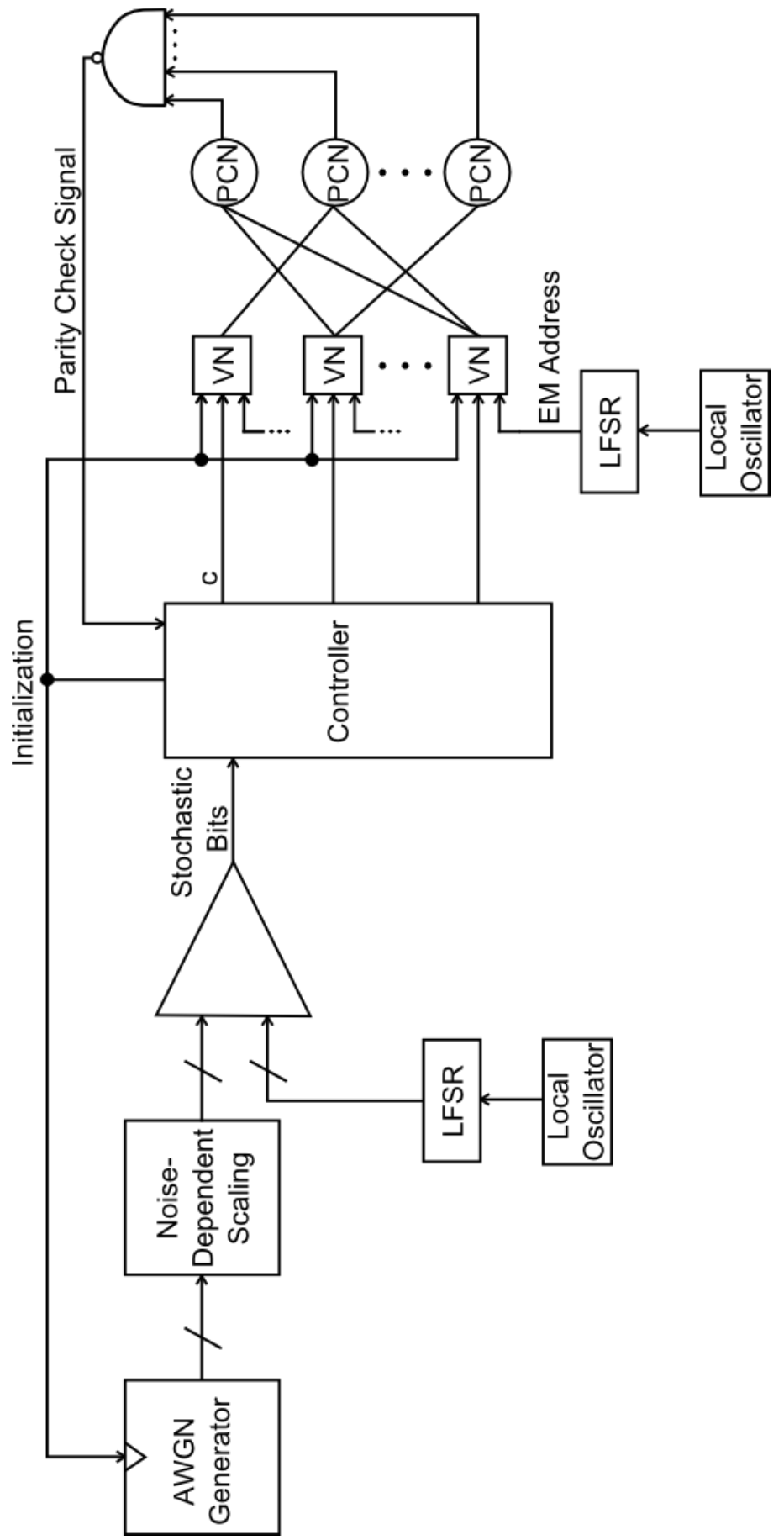

Figure 3.1: Block diagram of the clockless stochastic LDPC decoder. 


\subsection{AWGN Generator}

The decoder uses zero-value codewords with AWGN for testing purposes. The most common method of generating AWGN on-chip is using the Box-Muller transformation, shown below $[23,24]$.

$$
Y=\sqrt{-2 \ln \left(x_{1}\right)} \cos \left(2 \pi x_{2}\right)
$$

where $x_{1}$ and $x_{2}$ are two uniformly distributed random numbers and $Y$ is a normally distributed random number. To implement this algorithm in a digital circuit, two LUTs and a multiplier are required. In addition, the square root operation is very non-linear and therefore the quantization error of the LUTs will be large if the LUT uses linear indexing. To avoid this nonlinearity as well as significantly reduce the area consumption of the AWGN generator, a new design is proposed here.

An AWGN distribution can be generated using a single LUT by dividing the LUT into a set of bins with sizes calculated from the desired distribution. The Gaussian distribution is first calculated across upper and lower bounds $x_{U}$ and $x_{L}$ which are calculated using the following equation:

$$
x_{L, U}=\mu \pm \sigma \sqrt{-2 \ln (\beta)}
$$

where $\mu$ and $\sigma$ are the mean and standard deviation of the AWGN distribution and $\beta$ is the ratio of the probability at the outer bounds to the probability at the distribution center. In this design $\beta=0.01$ was used. The distribution is then divided into a number of bins. The bin counts are 
normalized and then used to partition the LUT. For example, the bin with the highest count, the peak of the distribution, will occupy the most space in the LUT. Therefore when a uniformly distributed random bit, such as that generated from an LFSR, is used as an input it will have a probability of falling on an address with the largest bin approximately equal to the probability of the bin calculated from the distribution. A hardware simulation of this design generating a Gaussian distribution with $\sigma=1$ and zero mean is shown in Figure 3.2 compared with a numerically generated distribution using the Box-Muller Algorithm and the ideal Gaussian distribution calculated using MATLAB.

Before being sent to the stochastic bit generators, the LLRs are first converted into channel probabilities using a separate 8-bit LUT which evaluates the following equation [11]:

$$
P_{i}=\frac{e^{\frac{L_{i}^{\prime}}{G}}}{e^{\frac{L_{i}^{\prime}}{G}}+1}
$$

$L_{i}^{\prime}$ is the scaled LLR and $P_{i}$ is the channel probability. $G$ is a scaling parameter which is adjusted based on the SNR to decrease the quantization error as the LLRs are sent between LUTs. 


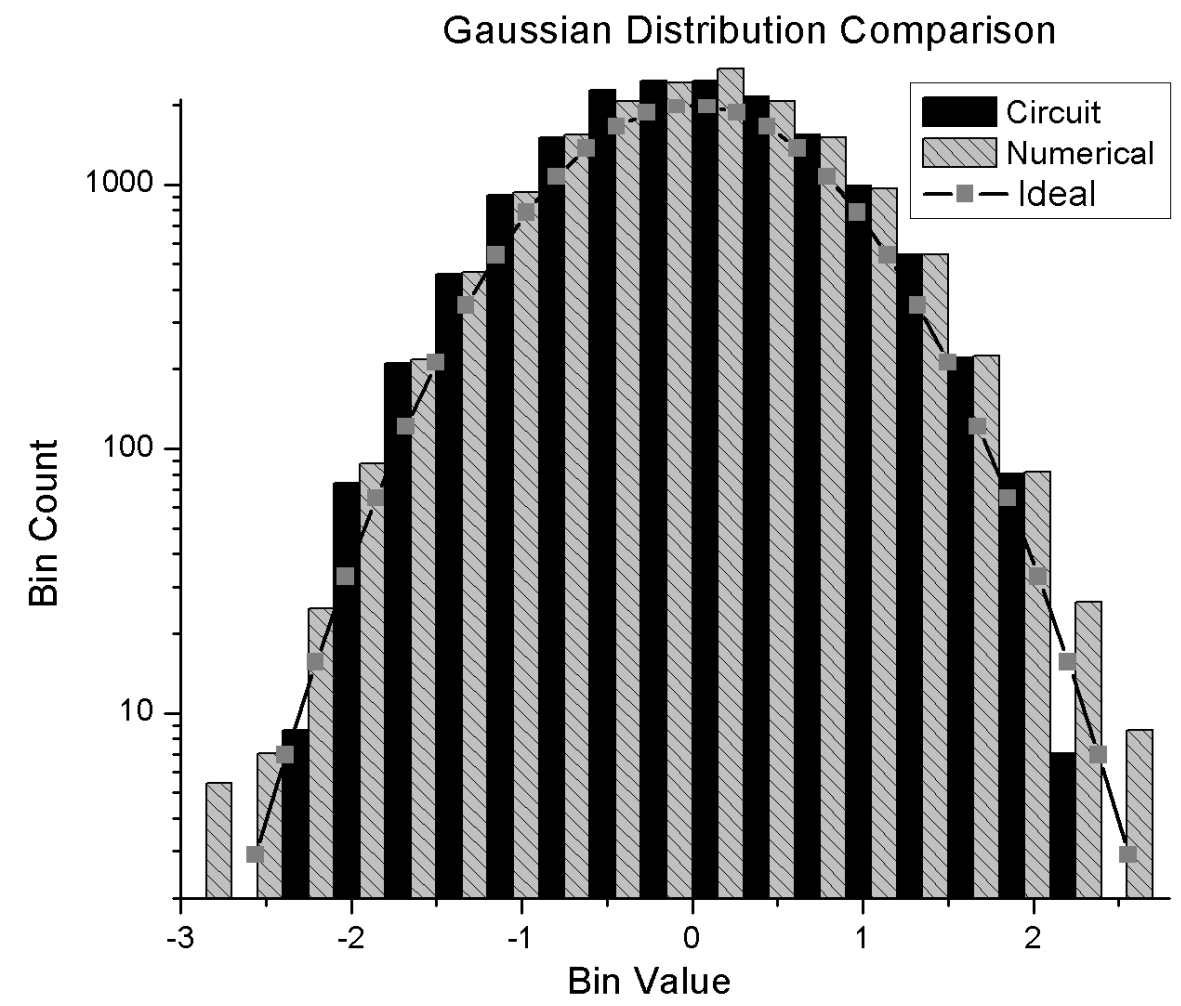

Figure 3.2: Comparison of the Gaussian distributions generated from a Box-Muller numerical simulation (shaded bars), the proposed Gaussian generation technique (black bars), and the scaled ideal probability distribution (line). The distributions are quantized to 8-bits, the same as the generator used in the decoder and are shown with a logarithmic scale. The distribution edges of the proposed AWGN generator could be improved by increasing the number of bins and/or the bit resolution. The Gaussian distribution has $\mu=0$ and $\sigma^{2}=0.5012$. The histogram plots were generated with a sample size of 18000 , roughly equivalent to 90 frames of the $(204,102)$ decoder, which is sufficiently large to illustrate the distribution shape. 


\subsection{Random Number Generator}

Random numbers are used by the AWGN generator, the stochastic comparator, and the generation of addresses for the EMs. These random numbers are generated using 16-bit LFSRs $[25,26]$. The LFSRs are constructed using the primitive polynomial $x^{16}+x^{5}+x^{3}+x^{2}+1$ which generates $\left(2^{16}-1\right)$ 16-bit random numbers (RNs) before repeating the cycle. The choice of a primitive polynomial configuration ensures that the LFSR produces a maximum length sequence. These RNs are then split into two 8-bit RNs which are used by the AWGN generator and the stochastic stream generators. Since the EMs are 8-bit registers they only require a 3-bit address and so the 8-bit EM RNs are further divided. Also, different EMs will have different stored bits and so the same address can be used for more than one EM. For a $(96,48)$ code with 96 variable nodes and 288 EMs (each node has three outputs) only 96 random addresses are used.

For high SNR testing where many frames are required, the decoder may need more than $\left(2^{16}-1\right)$ RNs in which case the RNs and the switching activity would repeat. To avoid this

problem, the LFSRs are reinitialized with new seeds when the decoder approaches $\left(2^{16}-1\right)$ RNs. If $K$ sets of seeds are stored in the decoder, this allows for $K\left(2^{16}-1\right)$ unique RNs. However, as $K$ increases, so does the FPGA logic utilization as well as the compilation time. To maximize the number of unique frames, the seeds are divided into two groups each with size $K / 2$. When all the seeds have been used they are reset back to the initial seeds but with one group offset by 
one. Therefore, the two groups will go through every combination before repeating themselves allowing for $K^{2}\left(2^{16}-1\right)$ unique RNs per LFSR. An illustration of this technique is shown in Figure 3.3.

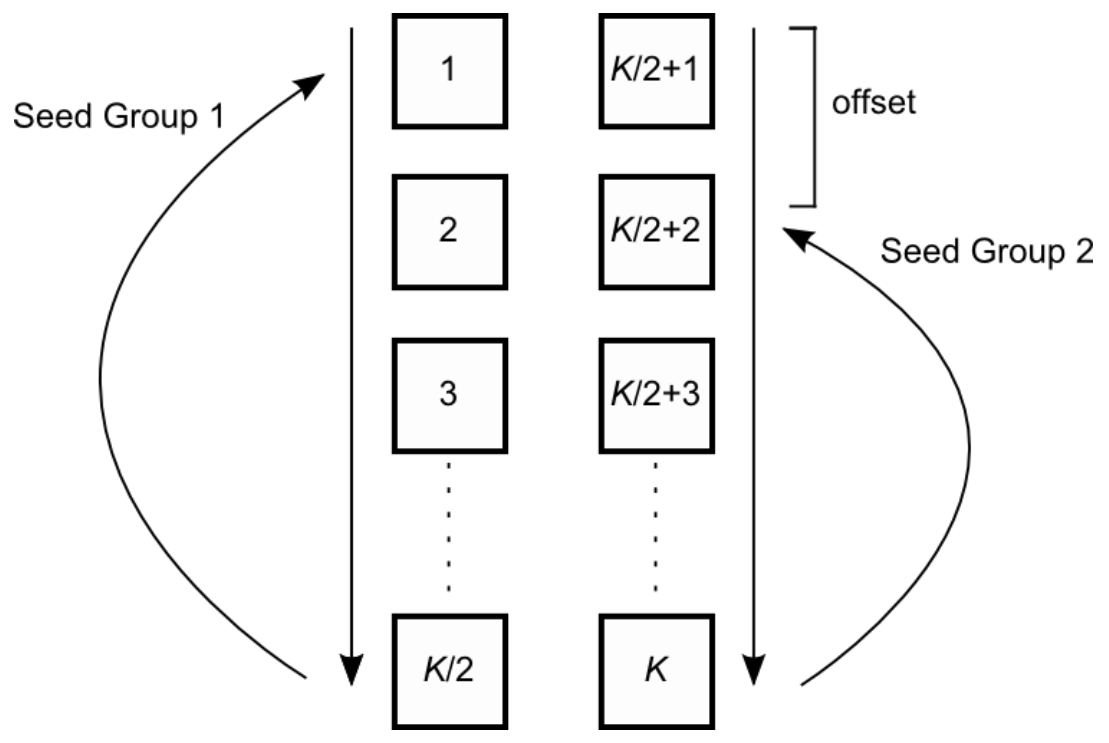

Figure 3.3: Illustration of the seed rotating technique. Each block represents a set of seeds used by LFSRs. Arrows represent which block of seeds is currently being used.

The stochastic comparator and the EM address generators are used throughout the decoding process while the AWGN generators are used only at the beginning of each frame. Therefore it is not necessary to update the LFSR seeds used for the AWGN generators. If the decoder exceeds $\left(2^{16}-1\right)$ frames and the channel probabilities repeat, the LFSRs used to generate stochastic bits and EM addresses will still be unique and so the switching activity of the decoder will also be different. This reduces the size of the AWGN generator module. 


\subsection{Stochastic Stream Generator}

Stochastic bits are generated using a comparator, shown in Figure 3.4, with two inputs: the 8-bit channel probability and an 8-bit random number generated from a LFSR. If the channel probability is larger than the random number a ' 1 ' is generated, otherwise the output is ' 0 '. The LFSR is triggered by a local oscillator such as a ring oscillator. The LFSR could also be triggered by a global clock since clock delays will not impact the decoder performance due to the partial-update algorithm.

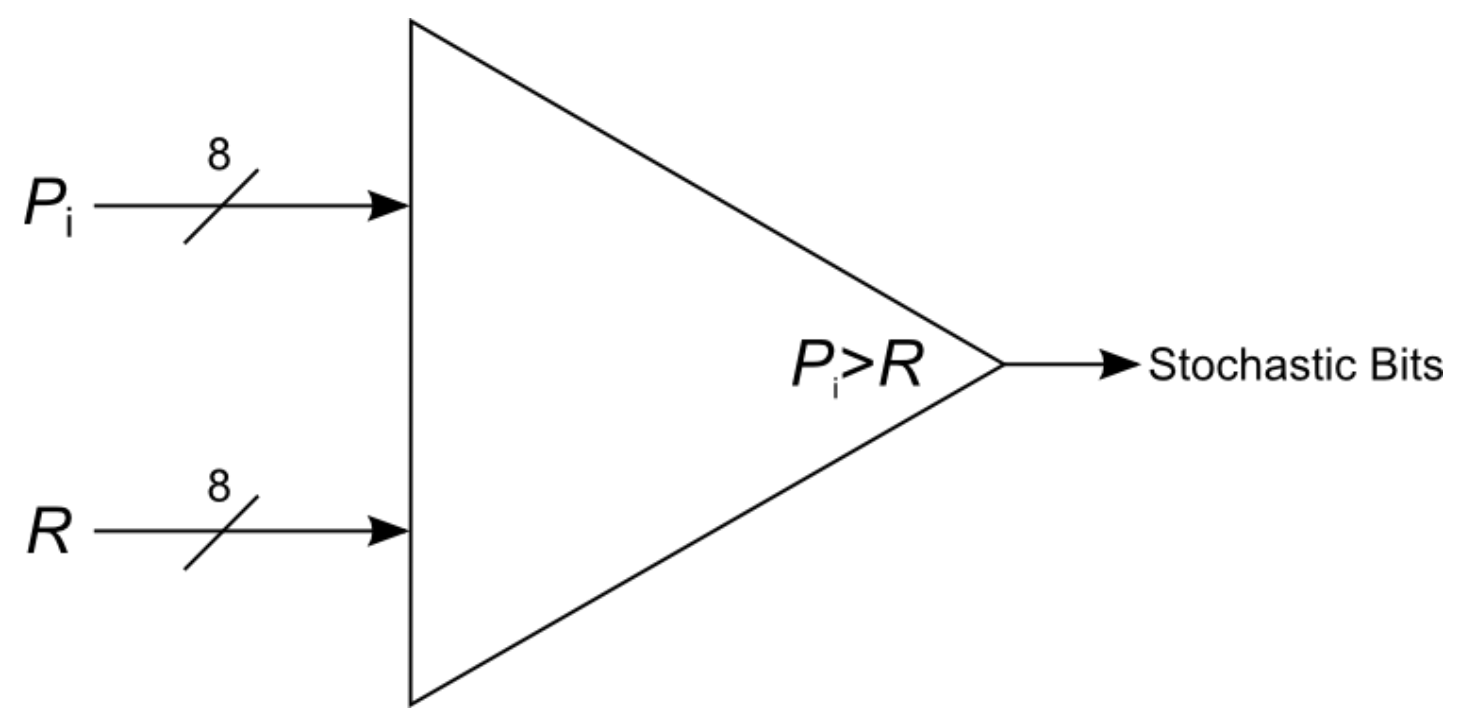

Figure 3.4: Comparator used for stochastic bit generation. The 8-bit inputs $P_{i}$ and $R$ are the channel probabilities and a random number. 


\subsection{Controller}

The controller routes stochastic bits from the comparators to the interleaver and controls the rotation of seeds for the (pseudo) random number generators. The controller also toggles the decoder between two main states:

- Initialization phase: During the initialization phase, the stochastic bits are fed directly into the EMs of the variable nodes, bypassing the node equations. During initialization, the signals between nodes are suppressed. The beginning of the initialization phase also triggers the AWGN generator to produce a new set of noise signal inputs. After a certain number of initialization phases have passed, the controller instructs the seed module to rotate to a new set and for the LFSRs to be reinitialized. Depending on the speed of stochastic bit generation, the initialization phase continues for as long as it takes to fill the EMs with channel probabilities.

- Decoding phase: Immediately after the EMs have been preloaded, the controller disables the initialization signal and the connection from the stochastic stream directly to the EMs is severed. The channel probabilities are instead set as inputs to the variable nodes. The connections between variable and parity-check nodes are allowed to communicate freely, limited only by their gate and wire delays. During the decoding phase the controller checks for the parity-check equations of each parity-check node to be satisfied simultaneously. When this occurs, a valid codeword has been found by the decoder and the initialization phase begins for the next decoding frame. While decoding the controller also increments a counter using the on-board 50MHz clock. When the counter reaches the predefined value 300 , an error is declared and the next 
frame is initialized. The value of this counting limit duration is chosen based on the desired performance trade-off between throughput and FER.

\subsection{Variable Nodes}

The 3-input continuous time variable node circuit is shown in Figure 3.5. This circuit was proposed in [15]. Signal ' $U$ ' determines whether the node is in a regenerative state (all inputs are equal) or a degenerative state. The variable node behavior is summarized in Table 3.1.

Table 3.1: Summary of the variable node behavior.

\begin{tabular}{|c|c|c|c|}
\hline Inputs & $\mathbf{U}$ & state & Output \\
\hline 000 & 1 & regenerative & 0 \\
\hline 001 & 0 & degenerative & EM \\
\hline 010 & 0 & degenerative & EM \\
\hline 011 & 0 & degenerative & EM \\
\hline 100 & 0 & degenerative & EM \\
\hline 101 & 0 & degenerative & EM \\
\hline 110 & 0 & degenerative & EM \\
\hline 111 & 1 & regenerative & 1 \\
\hline
\end{tabular}

While in a degenerative state the output of the node is selected from the EM from a random address generated by an LFSR. When the node changes to a regenerative state signal ' $\mathrm{U}$ ' generates a pulse triggering the EM to store the new regenerative bit. This bit is also used as the node output during a regenerative state. The EM consists of an 8-bit shift register with an output selectable using an address. While the initialization signal is ' 1 ', the channel probability bits are stored directly in the EM. In the FPGA implementation, a series of five inverters is used 
rather than the one shown in Figure 3.5. This lengthens the duration of the pulses to ensure that they are detected by the EM. The number of inverters was chosen by increasing the length of the inverter series until the EM detected the pulses reliably. The minimum number of inverters needed would is likely dependent on the routing techniques and the FPGA architecture. Note that a pulse is not generated in the event of transitioning from one regenerative state to another (from all ' 0 ' inputs to all ' 1 ' inputs or vice versa). Since the inputs are not synchronized, the probability of this happening is extremely small, so the gain in performance for detecting this transition would be small compared to the extra hardware required to detect a change in the inputs. Each variable node has three connections, three inputs and three outputs. Therefore, each node has three copies of the circuit in Figure 3.5. If one of these circuits has an output wire connected to some parity-check node ' $\mathrm{X}$ ', then the incoming signal from parity-check node ' $\mathrm{X}$ ' is excluded from the inputs of that particular circuit.

Since there is no synchronization block in the clockless variable node circuit, there is the potential for metastability. This phenomenon was investigated by Onizawa in [43] through timing simulations and it was concluded that the probability of metastibility is low and the effect on the error rate performance is minimal. This is due to the statistical nature of stochastic computing where small discrepancies have little effect on the statistical mean. A more detailed analysis of the metastability would require an investigation of how the FPGA implements specific components, but this is outside the scope of this thesis. 


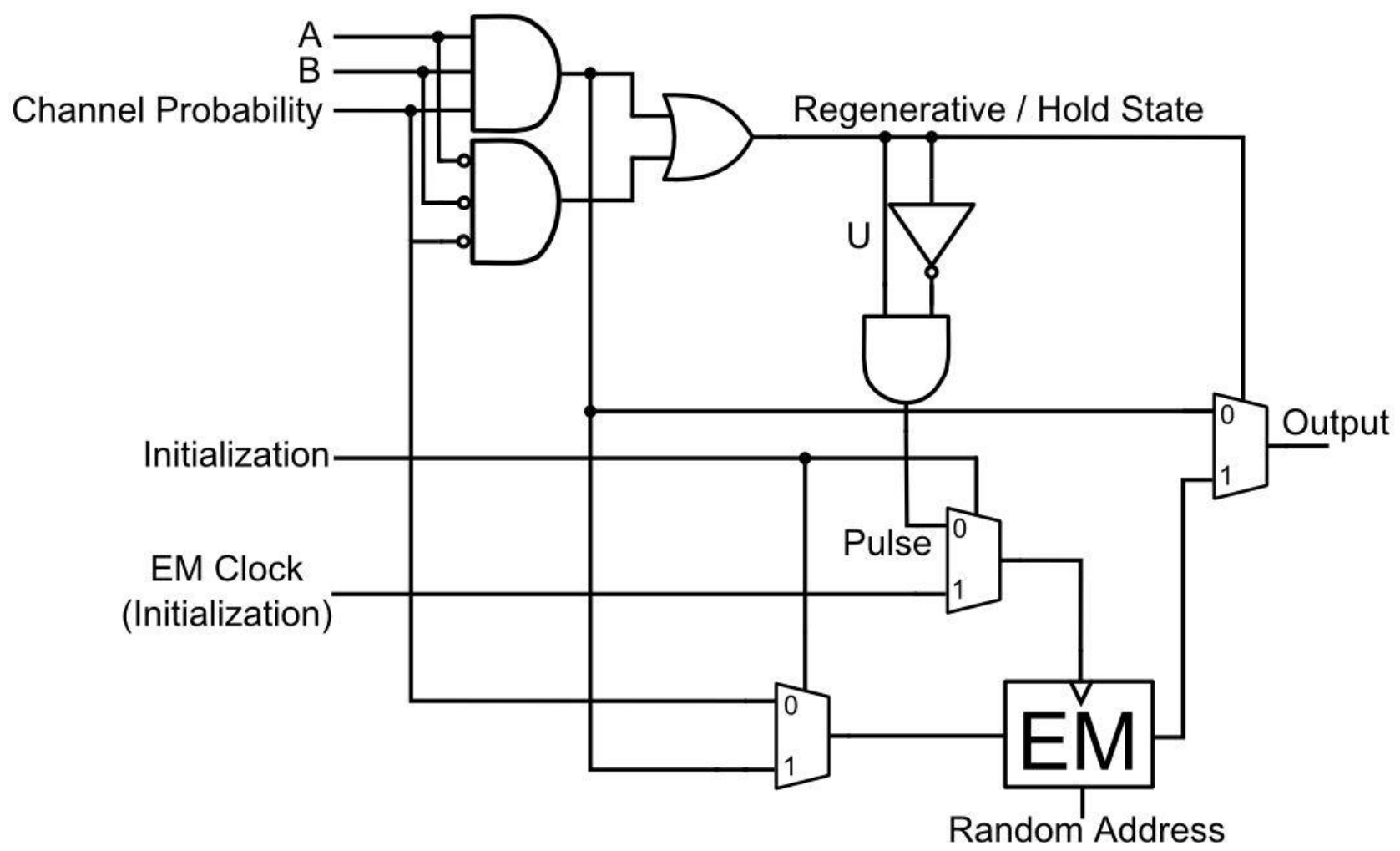

Figure 3.5: Circuit implementation of a 3-input clockless stochastic variable node using an edge memory (EM). Signal U is used to generate a pulse to trigger the EM. Although only one inverter is shown here, several are used to lengthen the duration of the pulse.

The EM, a schematic of which is shown in Figure 3.6, consists of an 8-bit pulse-triggered shift register. The output is taken from a multiplexor which randomly selects a bit stored in one of the flip-flops. 


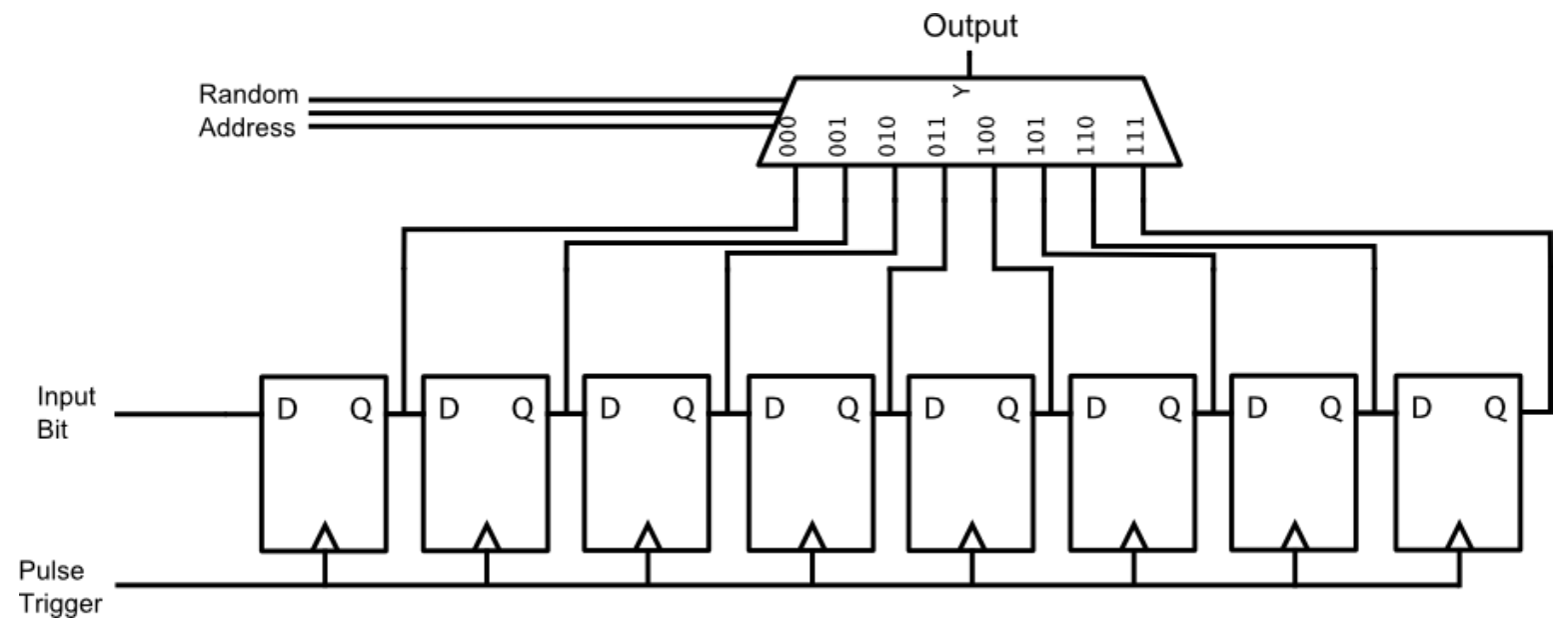

Figure 3.6: Schematic of the edge memory (EM) used in the clockless stochastic variable node.

\subsection{Parity-Check Nodes}

Previous implementations of stochastic LDPC decoders have used the parity-check node design shown in Figure 3.7 [28]. The red and blue lines represent the effect of a change in input ' $A$ ' on output ' 0 '. The red line illustrates a direct path from the input to the final XOR gate while the blue line passes through the 6-input XOR gate. The delay introduced by this extra XOR gate results in a glitch signal being generated after the red signal has reached the final XOR gate and the blue signal has yet to arrive. If the all of the parity-check nodes wait for each other to complete their operations, this does not present a problem. However, in a clockless interleaver this extra signal reaches the variable nodes and creates unexpected switching activity, decreasing the speed of convergence. 


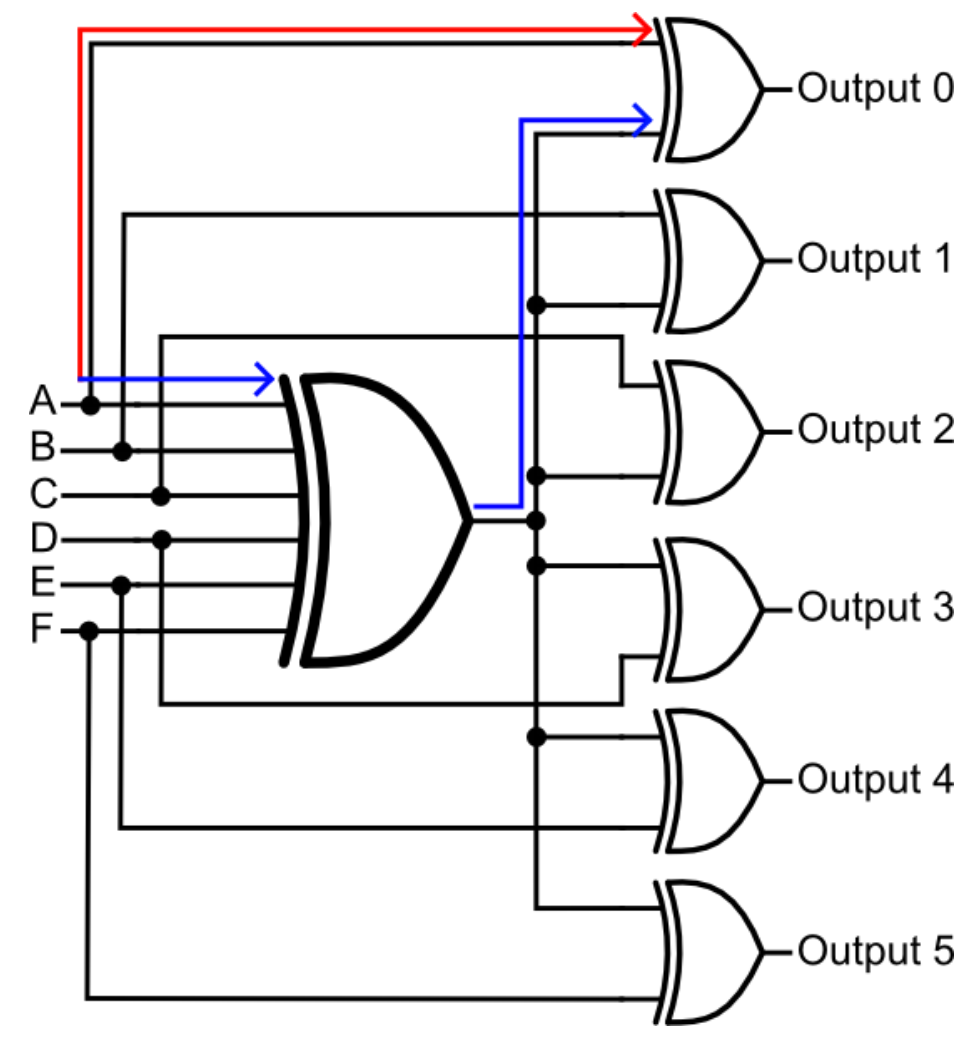

Figure 3.7: Parity-check node circuit for a synchronous stochastic LDPC decoder. The red and blue lines represent the paths from which input 'A' affects output ' 0 '.

With clockless, decoding all logical path delays must be equal since there is no mechanism to synchronize decoding cycles. The clockless parity-check node circuit used in this decoder, proposed in [15], is shown in Figure 3.8. 


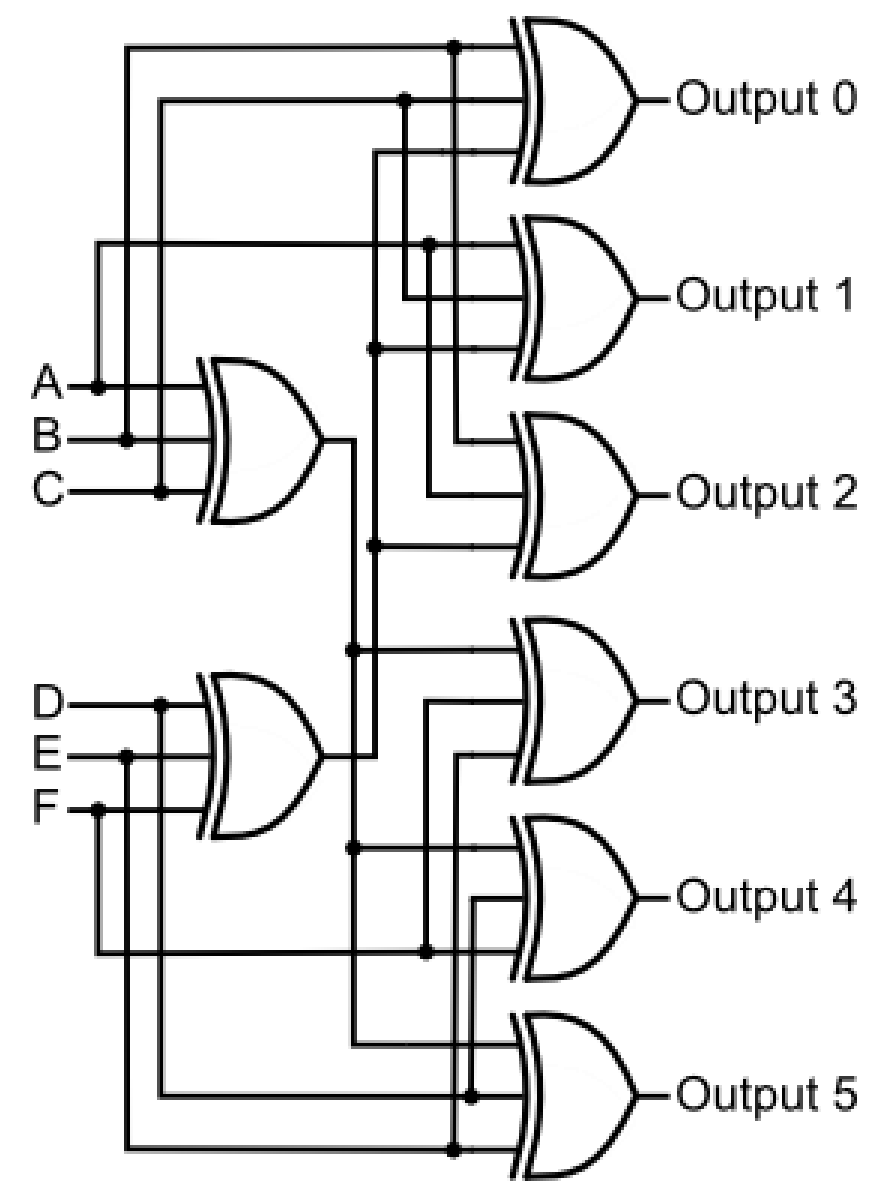

Figure 3.8: Circuit implementation of a 6-input clockless stochastic parity-check node.

In this design every input affects each output through only a single path. This eliminates the glitch signal at the cost of increased node complexity.

To determine if the decoder has reached a valid codeword each parity-check node must verify that all the inputs satisfy the parity check equations. In Figure 3.7 the parity check signal can be taken directly from the output of the 6-input XOR gate. In order for the controller to identify a valid codeword the parity-check equations are calculated from the output of the two 
front-end XOR gates with an additional 2-input XOR gate required to calculated the paritycheck equations for all the inputs. 


\section{Chapter 4}

\section{Results and Discussion}

This chapter reports the performance of the clockless stochastic LDPC decoder. Section 4.1 summarizes the FPGA logic utilization of the design. The frame error rate, throughput and power measurements are presented in Section 4.2, 4.3, and 4.4, respectively.

\subsection{Logic Utilization}

Table 4.1 shows the logic utilization of the $(96,48)$ and $(204,102)$ decoders while Figure 4.1 shows the chip layout of the FPGA with different modules highlighted. The stored seeds occupy a significant portion of the decoder to ensure unique random switching activity high SNR tests where many decoding frames are needed for a statistically significant number of errors. The section labeled "other" includes the control module, the LFSRs, the LUTs associated with the AWGN generator, and the comparators for stochastic bit generation. A larger $(408,204)$ decoder was also able to fit on the FPGA, however, due to the long compilation times this decoder was not tested extensively. 
Table 4.1: Summary of the logic utilization of the $(96,48)$ and $(204,102)$ stochastic clockless decoders synthesized on a ALTERA Stratix IV EP4SGX230 FPGA. These numbers refer to the total logic elements which is the sum of the total number of dedicated logic registers and total number of combinational functions.

\begin{tabular}{|c|c|c|c|c|}
\hline Decoder & \multicolumn{1}{c}{ Interleaver } & Seeds & Other & Total \\
\hline $\mathbf{( 9 6 , 4 8 )}$ & 6581 & 12836 & 13814 & $\begin{array}{c}33231 \\
(18 \%)\end{array}$ \\
\hline $\mathbf{( 2 0 4 , 1 0 2 )}$ & 11941 & 23291 & 25066 & $\begin{array}{c}60298 \\
(33 \%)\end{array}$ \\
\hline Available & & & & 182400 \\
\hline
\end{tabular}



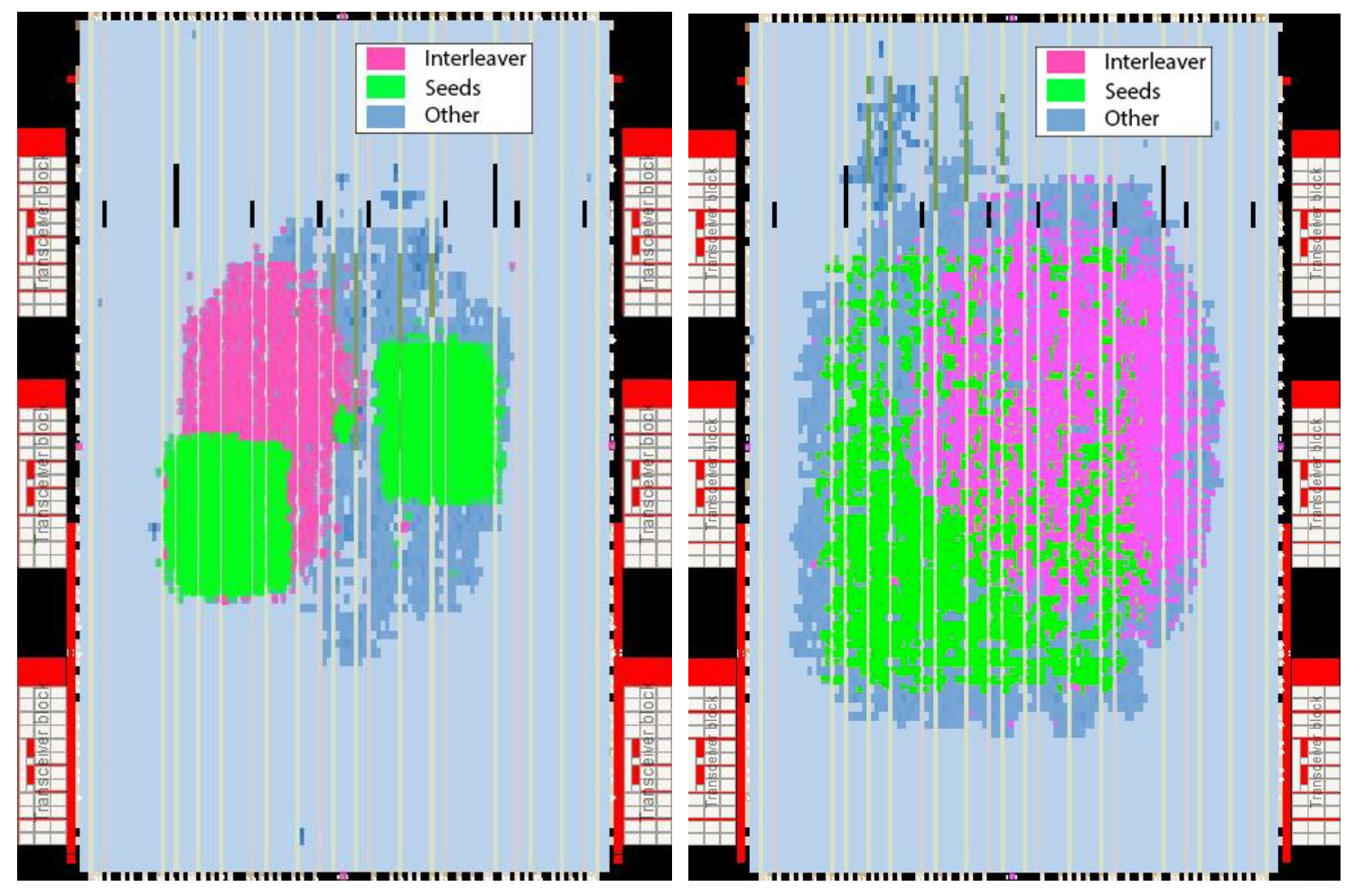

Figure 4.1: FPGA layout of $(96,48)$ decoder (left) and $(204,102)$ decoder (right).

Figure 4.2 and 4.3 show the estimated wire delays of the interleavers for the $(96,48)$ and the $(204,102)$ decoders. These delays were estimated using the TimeQuest Timing Analyzer [39] in Quartus II which determines the expected path delays between nets in the synthesized design. A tool command language (TCL) script was created to report the path delays from the variable to the parity-check nodes by specifying the start and destination pins. Since we are only interested in the direct paths from a variable to a parity-check node, a MATLAB script was used to eliminate the delay measurements which do not correspond to a connection in $\boldsymbol{H}$. 


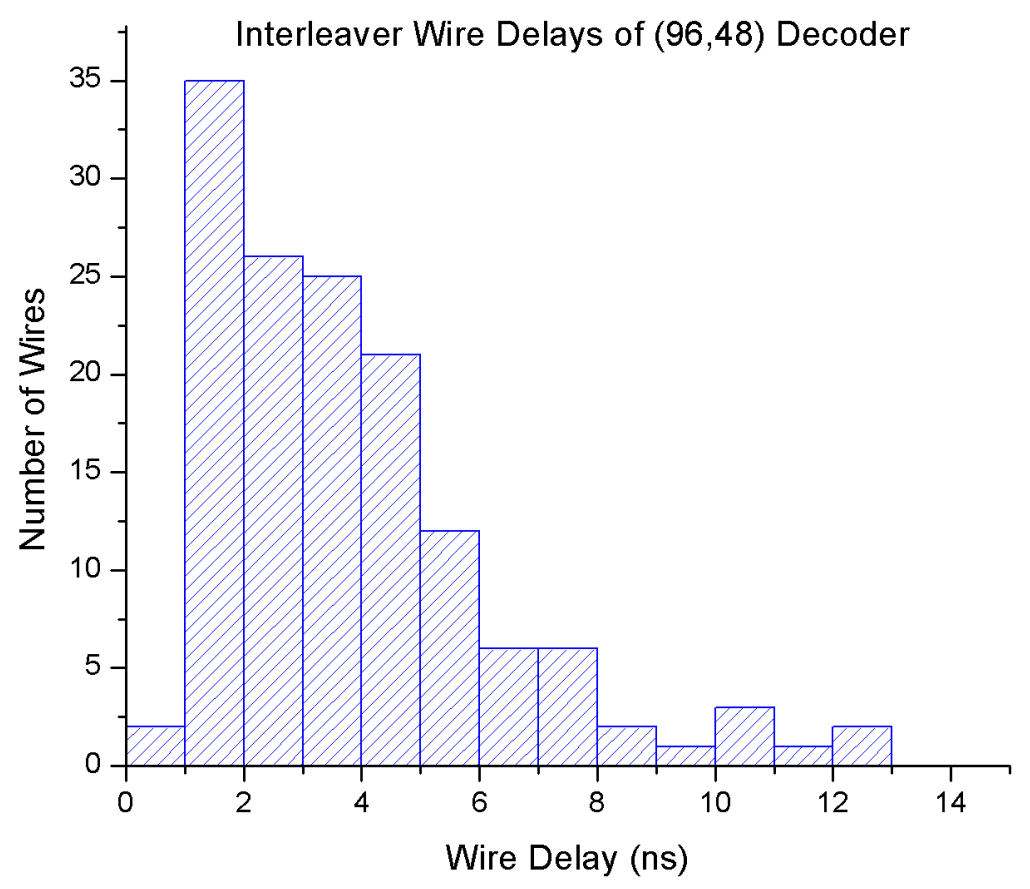

Figure 4.2: Distribution of interleaver wire delays of the $(96,48)$ decoder. Delays were determined by estimating the path delays of the synthesized design.

The largest wire delay from the $(96,48)$ decoder interleaver is $12.306 \mathrm{~ns}$. In a synchronous design, this would allow for a maximum clock frequency of $81.26 \mathrm{MHz}$. However, if we assume a clockless design where the limiting factor is the average wire delay, the equivalent clockless decoder could operate at 261.35 MHz. Similarly, the $(204,102)$ decoder would be limited to $96.28 \mathrm{MHz}$ while a clockless design would operate at $429.64 \mathrm{MHz}$. Note that this is maximum rate of switching activity in the decoder and not necessarily the 
throughput. That calculation would require an understanding of the decoding latency and the relationship between the number of iterations and the bit error rate.

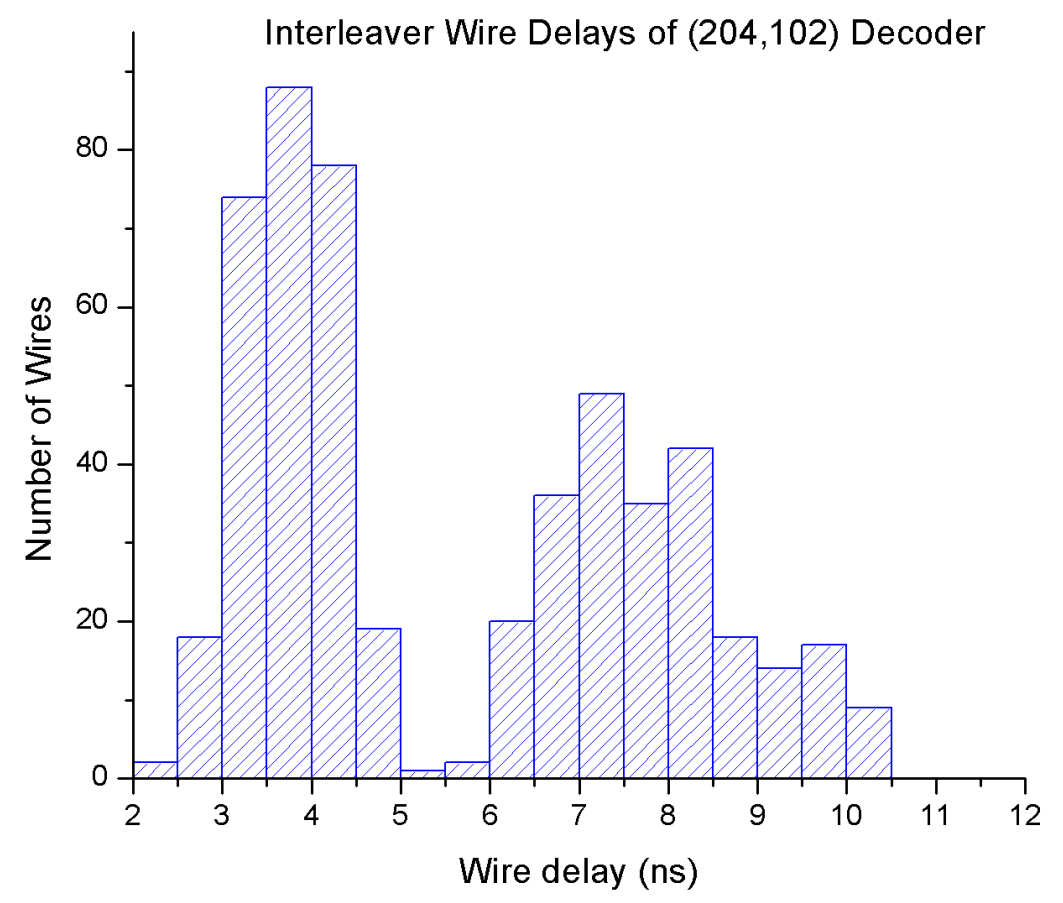

Figure 4.3: Distribution of interleaver wire delays of the (204,102) decoder. Delays were determined by estimating the path delays of the synthesized design. A possible explanation for the dual peaks is that the during the automatic fitting process some wires were routed using global routing, a routing technique used for wires spanning large quadrants of the chip, and some wires were routed using local routing, a technique used for smaller wires. Most wires routed using global routing would be significantly longer than those which used local routing. 


\subsection{Frame Error Rate}

The frame error rate (FER) is the ratio of the number of failed decoding frames to successful ones at a particular SNR. A decoding frame ends when a valid codeword is obtained and is identified by the parity-check nodes as satisfying all parity-check equations. The frame also ends when the decoding attempt is declared unsuccessful and an error is declared. In a synchronous or asynchronous decoder, an error is normally declared after a set number of decoding cycles, the number of signal exchanges between the variable and parity-check nodes. Since a clockless decoder operates using the partial-update algorithm where wire delays result in some connections communicating faster than others, there is no clear definition of a decoding cycle. Instead an error is declared after a counter reaches a predefined termination time, $\mathrm{T}_{\mathrm{e}}$. The longer this duration, the more time the decoder has to converge on a valid codeword and therefore the lower the FER. However, as $T_{e}$ increases, the throughput decreases. The FER of the $(96,48)$ decoder is shown in Figure 4.2 and the FER if the $(204,102)$ decoder in Figure 4.3. These are compared with numerical simulations of the Log-domain SPA of the decoders. The NDS parameter $a$ was chosen based on experimentation and value of $a=2$ was found to produce the best results. The parameter $Y$ was set to $Y=6$ for best results. The simulated FERs are obtained by evaluating Equations 2.7 to 2.11 for 50 iterations before declaring an error or until a valid codeword was obtained.

For a synchronous stochastic decoder, the bit error rate (BER) can be measured by calculating the hard decision bits using a simple up/down counter at each variable node. At the end of a decoding sequence, assuming a valid codeword has not been detected, each variable node will output a ' 1 ' if its counter is greater than zero or a ' 0 ' otherwise. This sequence of bits 
can be compared with the original message to determine the number of bit errors. For a clockless decoder, a time averaging circuit would be required at each variable node since there are no clock cycles to trigger a counter. For the purposes of this thesis, it was decided that the FER was sufficient for demonstrating the proof-of-concept decoder. 


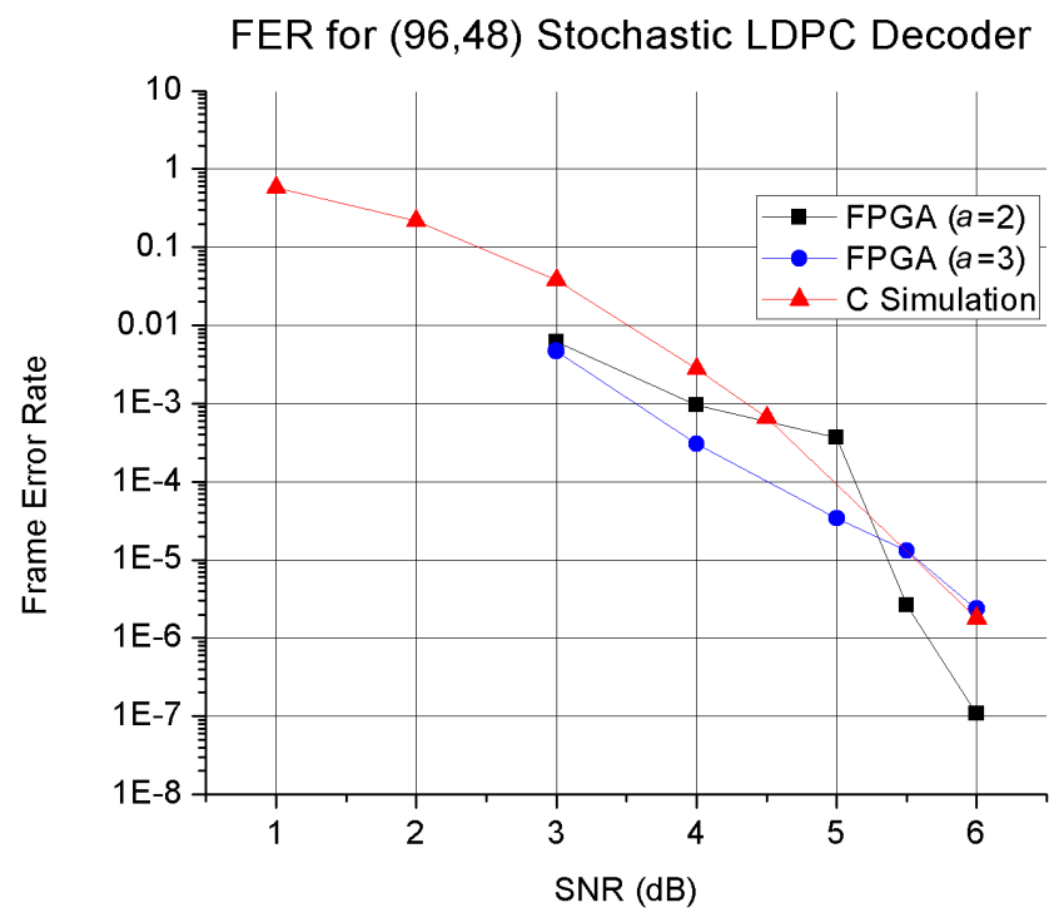

Figure 4.4: Frame error rate (FER) measurements of the $(96,48)$ clockless stochastic decoder implemented on an FPGA. Results are shown with noise-dependent scaling parameters $a=2$ and $a=3$. Measurements are compared with a numerical decoding simulation. 
FER for $(204,102)$ Stochastic LDPC Decoder

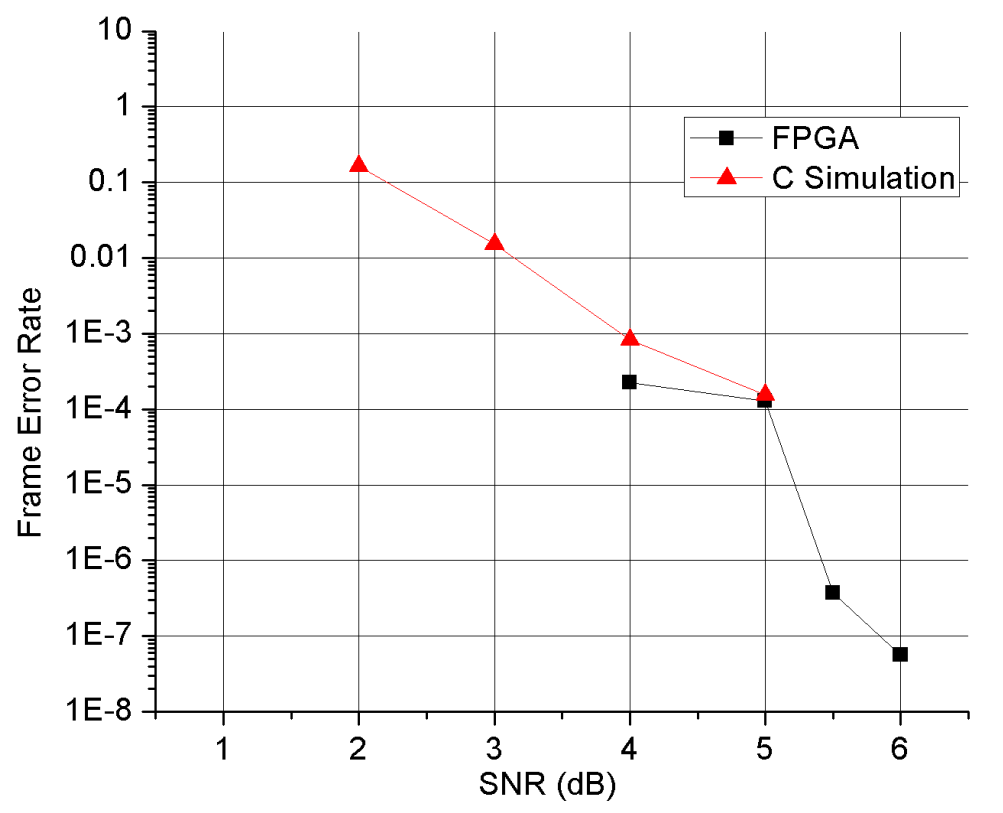

Figure 4.5: Frame error rate (FER) measurements of the $(96,48)$ clockless stochastic decoder implemented on an FPGA. Results are shown with noise-dependent scaling parameter $a=2$. Measurements are compared with a numerical decoding simulation.

\subsection{Throughput}

The throughput is the rate at which bits are decoded. As discussed earlier, the throughput depends heavily on the choice of $\mathrm{T}_{\mathrm{e}}$ as that affects the duration of each decoding frame. Figure 4.4 shows the throughput for the $(96,48)$ decoder and the $(204,102)$ decoder. As expected, the 
throughput is lower at low BERs since a longer duration of switching activity is required to reach a valid codeword.

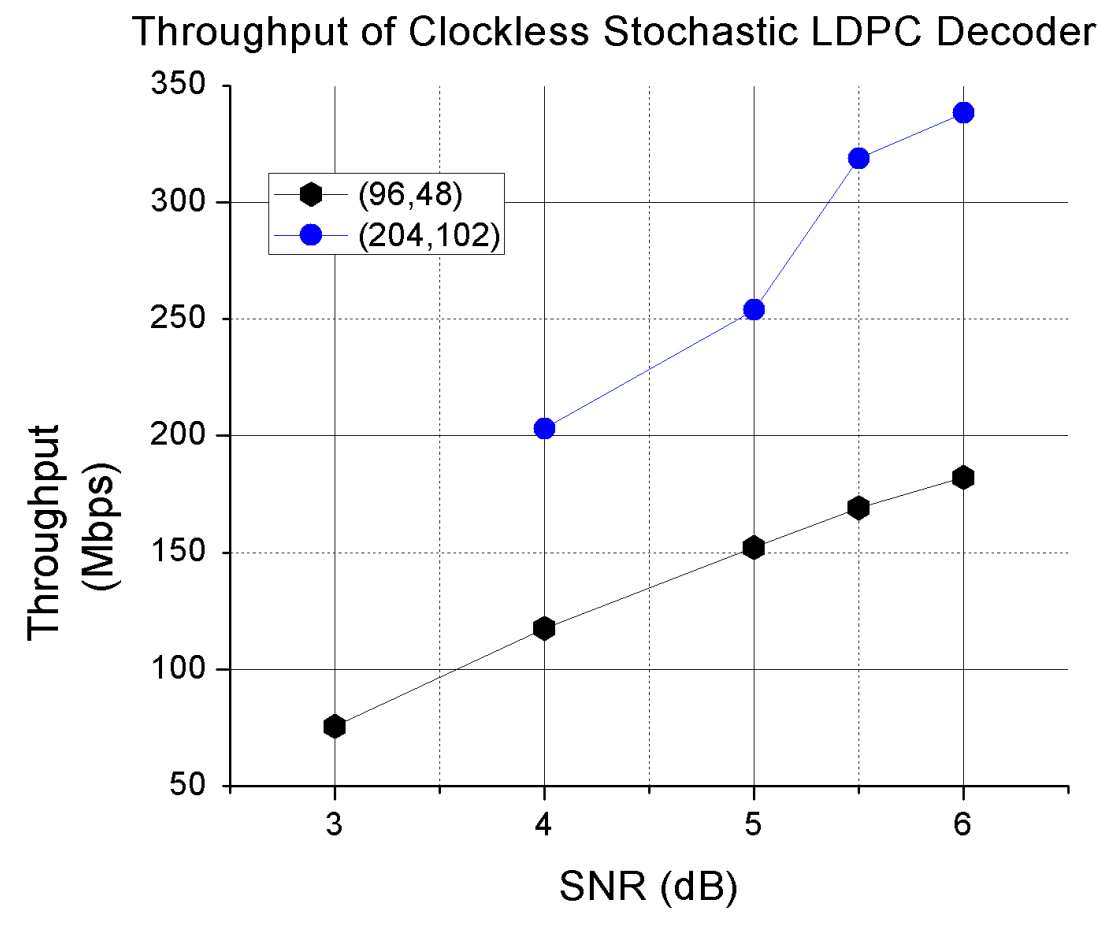

Figure 4.6: Coded throughput measurements of the $(96,48)$ and $(204,102)$ clockless stochastic decoders implemented on an FPGA. 


\subsection{Power Measurements}

Since the throughput varies at different SNRs, so does the power consumption of the decoder. The switching activity will also change, although this is minimized by the NDS. The power consumptions of the $(96,48)$ and $(204,102)$ decoders are shown in Figure 4.5. The FPGA power measurements were made using the technique developed by former MASc student Joyce $\mathrm{Li}$ in [34]. Although Quartus II contains a module for estimating the power consumption based on timing simulations, this technique has a large error [35]. The method used for power measurements in this thesis measures the power delivered to the FPGA directly using a voltmeter and a pair of $0.01 \Omega$ resistors. This power measurement is then subtracted from a similar measurement of the FPGA programmed with minimal logic. More details of the power measurement technique can be found in [34]. 


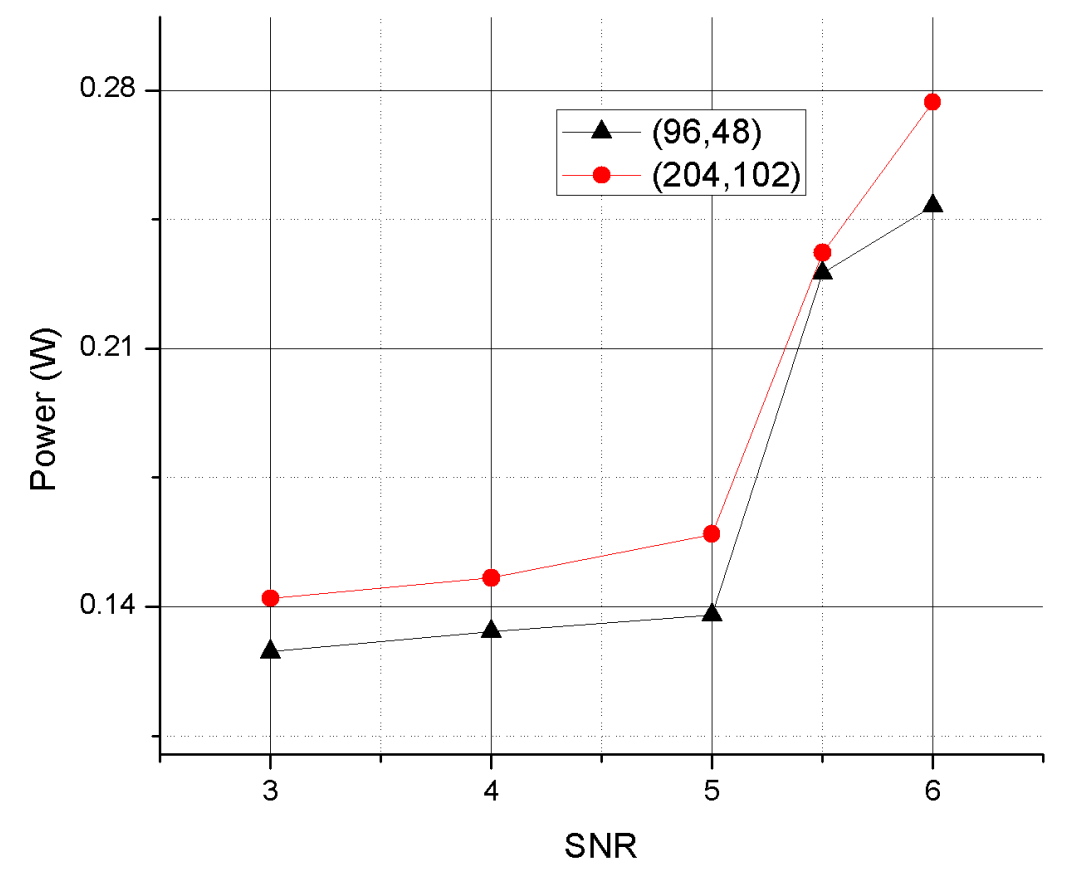

Figure 4.7: Power consumption of $(96,48)$ and $(204,102)$ decoders implemented on an FPGA.

The energy/bit of each decoder is shown in Figure 4.8, determined by dividing the power consumption by the throughput. 


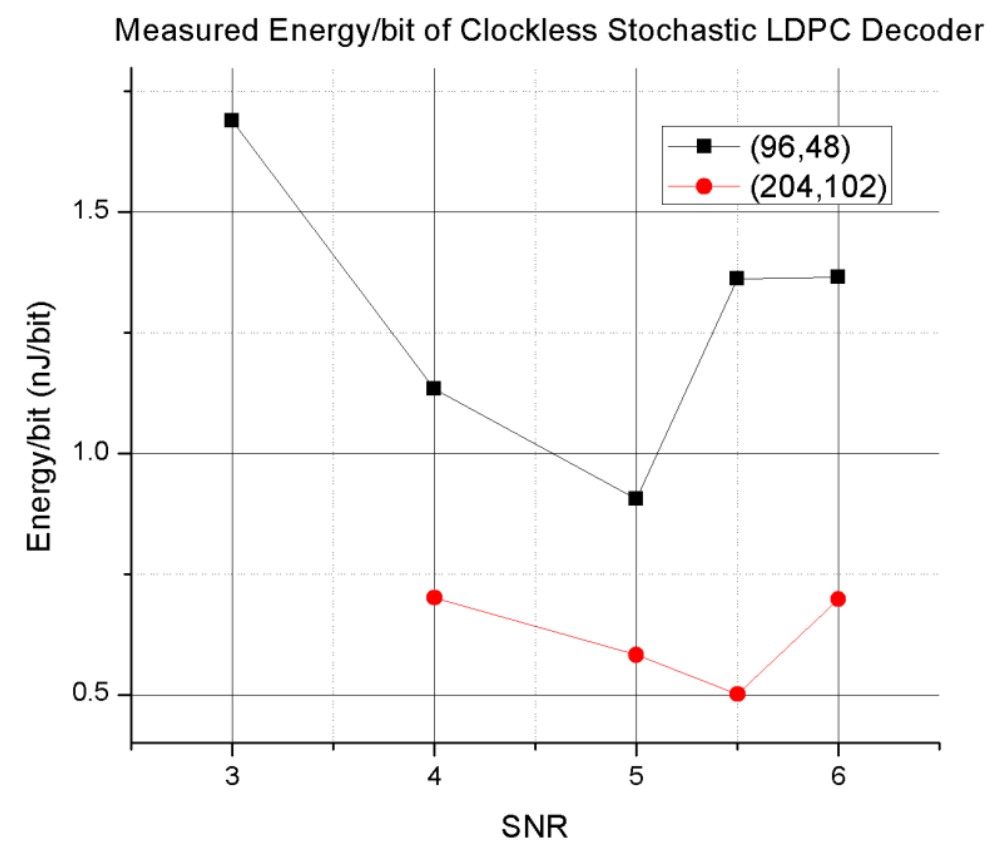

Figure 4.8: Energy-per-coded bit of $(96,48)$ and $(204,102)$ decoders implemented on an FPGA.

The results shown in this chapter have demonstrated that the implementation of the clockless stochastic LDPC decoder was successful. The $(96,48)$ and $(204,102)$ decoders here are not a large as what a practical decoder would use, and a larger code would have a higher throughput and would likely operate in the $\mathrm{GHz}$ range.

The $(204,104)$ decoder has a coded energy-per-bit of $0.7 \mathrm{~nJ}$ at $6 \mathrm{~dB}$. While this is not as small as the LDPC decoder implementations in [34], the purpose of the design was to build a proofof-concept design and not to perform power optimization. 


\section{Chapter 5}

\section{Conclusion}

The advantages of stochastic LDPC decoders include lower wiring complexity and lower power consumption. However, due to the serial nature of stochastic computing the throughput is limited by the clock speed. A clockless implementation of this type of decoder eliminates this limitation by allowing node computations to be completed purely through combinational logic. In this thesis research, an implementation of a clockless stochastic LDPC decoder was implemented on silicon. To our knowledge, this is the first implementation of its kind on an FPGA.

The results reported in Chapter 4 show that the clockless stochastic LDPC was successfully implemented on the FPGA. The clockless interleaver design eliminates the large high-speed clock network present in synchronous decoders reducing the limitations that the wire delays place on the throughput.

The HDL files for the decoder were generated using a C script and the FPGA was programmed using Quartus II. The variable and check-nodes were designed to operate in continuous time without the synchronization of node calculations. To reduce the noise floor and increase the speed of convergence, EMs and NDS were employed to increase the switching 
activity and therefore the speed of convergence. The large amount of random numbers required for the AWGN generator and stochastic bit generation were produced using LFSRs with a seed rotating technique that ensured that the decoder could evaluate many frames before repeating random numbers.

The scaling parameters here were chosen based on optimal performance through experimentation. However, a more detailed analysis of the error floor of this decoder is necessary for a quantitative determination of the optimal parameter choices. This type of analysis could also be used to predict the optimal clock speed for the generation of noise channel stochastic bits and for EM addresses.

LDPC decoders suffer from error floors at high SNR. It is possible that continuous time implementations will help to alleviate this issue in the future. 


\section{References}

1 R. G. Gallager. Low Density Parity Check Codes. Cambridge, MA: MIT Press, 1963.

2 R. G. Gallager, "Low density parity check codes," IRE Transactions Information Theory, Jan. 1962, 8, (1), pp. 21-28.

3 C. Berrou, A. Glavieux, P. Thitimajshima, "Near Shannon limit error-correcting coding and decoding: Turbo-codes," IEEE International Conference on Communications, Geneva. Technical Program, Conference Record, May 1993, 2, pp. 1064-1070.

4 D. MacKay and R. Neal, "Near Shannon limit performance of low density parity check codes," Electronics Letters, Aug. 1996, 32, (18), pp. 1645.

5 D. MacKay, "Good error-correcting codes based on very sparse matrices," IEEE Transactions on Information Theory, Mar. 1999, 45, (2), pp. 399-431.

6 C. Schlegel and L. Perez, Trellis and Turbo Coding. Piscataway, NJ: IEEE Press, 2004.

7 N. Traore, S. Kant, T. L. Jensen, (2007). Message Passing Algorithm and Linear Programming Decoding for LDPC and Linear Block Codes. Unpublished project, Aalborg University, Aalborg, Denmark. 
8 M. R. Islam, D. S. Shafiullah, M. M. A. Faisal, I. Rahman, "Optimized Min-Sum Decoding Algorithm for Low Density Parity Check Codes," International Journal of Advanced Computer Science and Applications, 2011, 2, (12), pp. 168-174.

9 D. Haley, A. Grant, J. Buetefuer, "Iterative Encoding of Low-Density Parity-Check Code," IEEE Global Telecommunications Conference, Nov. 2002, 2, pp. 1289-1293.

10 R. M. Tanner, "A recursive approach to low complexity codes," IEEE Transactions on Information Theory, Sept. 1981, 27, (5), pp. 533-547.

11 N. Onizawa, T. Hanyu, and V. Gaudet, "Design of high-throughput fully-parallel LDPC decoders based on wire partitioning," IEEE Trans.Very Large Scale Integr. (VLSI) Syst., 18, (3), pp. 482-489, Mar. 2010.

12 N. Onizawa, V. C. Gaudet, T. Hanyu, W. J. Gross, "Asynchronous Stochastic Decoding of Low-Density Parity-Check Code," IEEE 42nd International Symposium on MultipleValued Logic, May 2012, pp. 92-97.

13 N. Onizawa, W. J. Gross, T. Hanyu, V. C. Gaudet, "Lowering Error Floors in Stochastic Decoding of LDPC Codes Based on Wire-Delay Dependent Asynchronous Updating," IEEE 43rd International Symposium on Multiple-Valued Logic, May 2013, pp. 254-259.

14 N. Onizawa, W. J. Gross, T. Hanyu, V. C. Gaudet, " Low-energy asynchronous interleaver for clockless fully parallel LDPC decoding." IEEE Transactions on Circuits and Systems I: Regular Papers, Aug. 2011, 58, (8), pp. 1933-1943.

15 N. Onizawa, W. J. Gross, T. Hanyu, V. C. Gaudet, "Clockless stochastic decoding of low-density parity-check codes," IEEE Workshop on Signal Processing Systems, Oct. 2012, pp. 143-148. 
16 F. R. Kschischang, B. J. Frey, H. A. Loeliger, " Factor Graphs and the Sum-Product Algorithm," IEEE Transaction on Information Theory, 2001, 47, (2), pp.498-519.

17 V. K. Mansinghka, E. M. Jonas, and J. B. Tenenbaum, Computer Science and Artificial Intelligence Laboratory Technical Report, Stochastic Digital Circuits for Probabilistic Inference. Accessed February 13, 2014 [Online].

18 V. K. Mansinghka, E. M. Jonas, and J. B. Tenenbaum, Computer Science and Artificial Intelligence Laboratory Technical Report, Stochastic Digital Circuits for Probabilistic Inference. Accessed February 13, 2014 [Online]. Available: http://dspace.mit.edu/bitstream/handle/1721.1/43712/MIT-CSAIL-TR-2008069.pdf.

19 N. Onizawa, T. Hanyu, and V. Gaudet, "High-throughput bit-serial LDPC decoder LSI based on multiple-valued asynchronous interleaving," IEICE Trans. Electron., E92-C, (6), pp. 867-874, Jun. 2009.

20 N. Onizawa, T. Ikeda, T. Hanyu, and V. C. Gaudet, "3.2-Gb/s 1024-brate-1/2 LDPC decoder chip using a flooding-type update-schedule algorithm," Proc. IEEE Midwest Symp. Circuits and Systems, Aug. 2007, pp. 217-220.

21 N. Onizawa, T. Hanyu, and V. Gaudet, "Design of high-throughput fully-parallel LDPC decoders based on wire partitioning," IEEE Trans.Very Large Scale Integr. (VLSI) Syst., 18, (3), pp. 482-489, Mar. 2010.

22 D. J. C. MacKay. Encyclopedia of Sparse Graph Codes, http://www.inference.phy.cam.ac.uk/mackay/codes/data.html. 
23 E. Boutillon, J. Danger, A. Ghazel, H. Laamari, "Efficent FPGA implementeation of Gaussian noise generator for communication channel emulation," 7th IEEE International Conference on Electronics, Circuits \& Systems, 2000, 1, pp. 366-369.

24 Y. Fan, Z. Zilic, "A novel scheme of implementing high speed AWGN communication channel emulators in FPGAs," Proceedings of the 2004 International Symposium on proceeding of Circuits and Systems, 2004, 2.

25 P. Alfke, XILINX, Efficient Shift Registers, LFSR Counter and Long Pseudo-Random Sequence Generators. Accessed March 27, 2014 [Online]. Available: http://www.xilinx.com/support/documentation/application_notes/xapp052.pdf.

26 Maxim integrated, Pseudo-Random Number Generation touting for the MAX765x Microprocessor. Accessed January 21, 2014 [Online]. Available: http://www.maximintegrated.com/app-notes/index.mvp/id/1743.

27 S. S. Tehrani, S. Mannor, W. J. Gross, "Fully Parallel Stochastic LDPC Decoders," IEEE Transactions on Signal Processing, Oct. 2008, 56, 11, pp. 5692-5703.

28 K. Huang, V. Gaudet, M. Salehi, “A Markov chain model for Edge Memories in stochastic decoding of LDPC codes," 2011 45th Annual Conference on Information Sciences and Systems (CISS), March 2011, pp. 1-4.

29 W.J. Gross, V.C. Gaudet, A. Milner, "Stochastic Implementation of LDPC Decoders," Conference Record of the Thirty-Ninth Asilomar Conference on Signals, Systems and Computers, Oct. 2005, pp. 713-717.

30 C. Winstead, V.C. Gaudet, A. Rapley, C. Schlegel, "Stochastic iterative decoders," Proceedings. International Symposium on Information Theory, Sept. 2005, pp. 11161120. 
31 S. S. Tehrani, W. J. Gross, S. Mannor, "Stochastic decoding of LDPC codes, ” IEEE Communications Letters, Oct. 2006, 10, 10, pp. 716-718.

32 S. S. Tehrani, S. Mannor, W.J Gross, "An Area-Efficient FPGA-Based Architecture for Fully-Parallel Stochastic LDPC Decoding," IEEE Workshop on Signal Processing Systems, Oct. 2007, pp. 255-260.

33 Tehrani S. S. (2011). Stochastic Decoding of Low-Density Parity-Check Coder. Unpublished doctoral dissertation, University of Waterloo, Waterloo, Ontario, Canada.

34 S. Li (2012). Power Characterization of a Gbit/s FPGA Convolutional LDPC Decoder. Unpublished Master of Applied Science dissertation, University of Waterloo, Waterloo, Ontario, Canada.

35 Quartus II Handbook, version 13.1. Accessed March 27, 2014 [Online]. Available: http://www.altera.com/literature/hb/qts/quartusii_handbook.pdf.

36 ALTERA Stratix IV Device Handbook, Volume 1. Accessed Match 14, 2014 [Online]. Available: http://www.altera.com/literature/hb/stratix-iv/stx4_5v1.pdf.

37 S. J. Johnson, Introducing Low-Density Parity-Check Codes. Accessed October, 2013 [Online]. Available: http://sigpromu.org/sarah/SJohnsonLDPCintro.pdf.

38 W. E. Ryan, An Introduction to LDPC Codes. Accessed October, 2013 [Online]. Available: http://tuk88.free.fr/LDPC/ldpcchap.pdf.

39 ALTERA The Quartus II TimeQuest Timing Analyzer, Volume 3. Accessed May 20, 2014 [Online]. Available: http://www.altera.com/literature/hb/qts/qts_qii53018.pdf. 
40 J. Von Neumann, "Probabilistic logics and the synthesis of reliable organisms from unreliable components," Automata studies 34, 1956, pp. 43-98.

41 V.C. Gaudet, A. Rapley, "Iterative decoding using stochastic computation," Electronics Letters, Feb. 2003, 39, (3), pp. 299-301.

42 M.P.C. Fossorier, M. Mihaljevic, H. Imai, "Reduced complexity iterative decoding of Low-Density Parity Check codes based on belief propagation," IEEE Transactions on Communications, May 1999, 47, (5), pp. 673-680.

43 N. Onizawa, W. J. Gross, T. Hanyu, V. C. Gaudet, "Clockless Stochastic Decoding of Low-Density Parity-Check Codes: Architecture and Simulation Model," Journal of Signal Processing Systems, Feb. 2013, ISBN: 1939-8018. 